\title{
PREDICCIÓN DE FRACASOS FINANCIEROS CON OPCIONES REALES BARRERA: UN ESTUDIO PARA EL MERCADO ARGENTINO
}

\section{FINANCIAL DISTRESS PREDICTION WITH KNOCK OUT REAL OPTIONS: A STUDY FOR THE ARGENTINE MARKET}

\author{
JEL Classification: G1, G33 \\ Received: July 8, 2019 | Accepted: February 26, 2020 | Available Online: February 28, 2020 \\ Cite this article as: Milanesi G. (2019). Predicción de fracasos financieros con opciones reales \\ barrera: Un estudio para el mercado argentino. Estudios de Administración, 26 (2), 52-81. \\ https://doi.org/10.5354/0719-0816.2019.56951
}

\section{Gastón Silverio Milanesi \\ Departamento Ciencias de la Administración, Universidad Nacional del Sur, Argentina Centro de Estudios para Análisis Financiero, Universidad de Buenos Aires, Facultad de Ciencias Económicas, Argentina \\ Universidad Tecnológica Nacional, Facultad Regional Bahía Blanca, Departamento de Licenciatura en Organización Industrial, Argentina milanesi@uns.edu.ar}

\section{Resumen}

El trabajo explora los modelos para la predicción de default basados en la teoría de opciones reales, proponiendo un modelo simple utilizando opciones exóticas barrera. Los modelos de predicción de fracasos financieros consideran el valor del patrimonio como una opción de compra sobre los activos de la empresa. Su principal debilidad reside en la relación directa entre que existe entre volatilidad del activo y valor de la opción. Las opciones barreras del tipo knock out ajustan inversamente la relación entre volatilidad y valor del patrimonio para determinados niveles críticos del activo. En esa línea, se propone una versión “naive” de modelo de opciones barrera para predecir default y calcular el valor esperado de quiebra de la empresa, sector y mercado. Primero se empleó el método de casos para realizar un estudio comparativo entre el modelo "naive” tradicional y las opciones barrera "naive”. Se aplicó el análisis de sensibilidad sobre variables como volatilidad, endeudamiento y horizonte de tiempo y se implementó sobre una muestra representativa de empresas del mercado argentino. Los resultados obtenidos indican el mejor desempeño del modelo de opciones barreras. Con aumentos de volatilidad el valor del capital tiende a disminuir, y presentó correlación directa entre volatilidad y probabilidad de default. El modelo es una herramienta útil para predecir probabilidades y valores de default de empresas en un nivel individual o agregado.

Palabras claves: Opciones Reales, Barreras, Default, Valor esperado Default 


\begin{abstract}
This paper explores default prediction models basis on real options theory, proposing a simple model using exotic barriers options. The financial distress prediction models consider the value of net capital like a call option over the firm's assets. Its main weakness resides in the direct relationship between asset's volatility and option value. The knock out barrier option type, for certain critical level of assets, adjusts inversely the relationship between volatility and net capital value. In this line, a naïve version of barrier models for predict financial distress and calculates default values for firms, sector and market is proposes. First, a case method is employed to make a comparative analysis between the traditional naïve model and the naïve barrier options. Over variables like volatility, leverage, time horizon, the sensitivity analysis is applied, and over a representative sample of Argentinian market' firms is implemented. The obtained results indicated the better development of the barriers option model. With increased volatility, net capital value tend to reduce, and showed direct correlation between volatility and default probabilities. The model is a useful tool to predict firms' probabilities and value of default, in an individual o aggregated level.
\end{abstract}

Keywords: Real options, Barriers, Default, Default expected value

\title{
1. Introducción
}

Black y Scholes; (1973) y Merton; (1974) fueron los primeros en afirmar que el valor del patrimonio de una empresa puede asimilarse a una opción de compra sobre los activos de la firma. Tal aseveración constituye la base conceptual de los modelos financieros basados la teoría de opciones ${ }^{1}$, diseñados para la predicción de fracasos financieros. En sus inicios adoptaron estructuras simples, evolucionando hacia formulaciones complejas mediante el uso de opciones exóticas barreras, del tipo knock out. A diferencia de las opciones simples, este tipo de modelo tiene la capacidad de reflejar el efecto negativo que presenta la excesiva volatilidad sobre el valor del patrimonio (Brockman y Turtle, 2003); (Reiz y Perlich, 2007). En tal sentido, el trabajo desarrolla e implementa una versión operativa del modelo adaptando las propuestas existentes en la literatura sobre opciones barrera (Brockman y Turtle, 2003; Reiz y Perlich, 2007; Milanesi, 2016 y Milanesi, Pesce, y El Alabi, 2016). El funcionamiento del modelo es ilustrado con un estudio de caso hipotético, aplicando análisis de sensibilidad de variables como volatilidad, plazo y endeudamiento. Seguidamente se desarrolló su implementación práctica y estudio del poder predictivo de los modelos. En primer término, vía análisis comparativo de la serie temporal de probabilidades de insolvencia correspondientes a dos empresas con desempeño financiero diferenciado. Luego se determinaron probabilidades y valor esperado de default, medidas propuestas

1 Complementando a los modelos estructurales o Z-score (Altman, 1968 y 1993; Altmant y Kishore, 1996 y Hillegeist, Keating, Cram, Lundstedt; 2004). Los modelos estructurales actualmente se complementa con modelos de análisis discriminante multivariado y modelos logit de regresión, validando el poder explicativo de los ratios de rentabilidad, solvencia y flujos de fondos (Smaranda, 2014) 
para monitorear la salud financiera de empresas, un sector o mercado en un todo. Las medidas se aplicaron sobre 53 empresas cotizantes en el mercado de capitales argentino, sobre el ejercicio 2018. Se obtuvieron resultados a nivel sectorial y global, corroborando las ventajas del modelo con opciones barrera, atendiendo al impacto de la volatilidad y variaciones en el endeudamiento.

\section{2- Opciones y modelos para predicción de fracasos financieros.}

En esta sección se desarrollan los principales modelos basados en la teoría de opciones.

\section{1-Los modelos Merton y KMV:}

Merton (1974) desarrolló el primer modelo de predicción de default, empleando el valor nominal de los pasivos, valor de mercado del patrimonio neto y la volatilidad de rendimientos de la acción. Se supone que el pasivo tiene una estructura de pagos equivalente a un bono cupón cero con vencimiento en $(T)$. El fracaso financiero acontece cuando, al vencimiento, el valor del activo $(A)$, es inferior al pasivo, por lo tanto $P N=\max \left(A_{T}-D ; 0\right)$ con;

$$
\begin{gathered}
P N=A_{0} N(d)-P e^{-r T} N\left(d-\sigma_{A} \sqrt{(T)}\right)(1) \\
d=\frac{\ln \left(A_{0} / P\right)+\left(r+\frac{\sigma_{A}^{2}}{2}\right)(T)}{\sigma_{A} \sqrt{ }(T)}
\end{gathered}
$$

Donde $(r)$ y $N($.$) representan la tasa libre de riesgo y la distribución normal estándar$ acumulada. Jones, Masson y Rosenfeld, (1984) proponen una sencilla relación para obtener la volatilidad del activo $\left(\sigma_{A}\right)$ a partir de la volatilidad del patrimonio $\left(\sigma_{P N}\right)$,

$$
\sigma_{P N}=\frac{A}{P N} N(d)\left(\sigma_{A}\right)(3)
$$

Resolviendo simultáneamente es sistema de ecuaciones 1 y 3 es obtenida la volatilidad del activo, luego es calculada la distancia de default $(D D)$,

$$
D D=\frac{\ln \left(A_{0} / P\right)+\left(R O A-\frac{\sigma_{A}^{2}}{2}\right)(T)}{\sigma_{A} \sqrt{ }(T)}(4)
$$

La pendiente de crecimiento del activo es ${ }^{2} \mu_{A}$ que en términos operativos es calculada mediante el rendimiento observado sobre los activos (ROA). La probabilidad de default $(P D)$ es,

$$
P D=N(-D D)(5)
$$

Del modelo anterior se deriva el conocido modelo iterativo $K M V^{3}$. Este se caracteriza por calcular iterativamente el valor diario del activo con la estimación de la serie diaria de volatilidad $\left(\sigma_{A}\right)$, obteniéndose $\sigma_{A}^{K M V}$ y $\mu_{A}{ }^{K M V}$. Las variables se

2 Pendiente y volatilidad se suponen constantes. Al utilizarse $\mu_{A}$ en lugar de la tasa libre de riesgo, se obtienen las probabilidades reales u objetivas.

3 Se lo conoce como Moodys KMV desarrollado en la división de riesgo de Moody, siendo sus autores Stephen Kealhofer, John Mac Quown y Oldrich Vasicek. (Vasicek, 1984, 2001); (Crosbie y Bohn, 2002), (Hillegeist, Keating, Cram, Lundstedt, 2004), 
incorporan en la ecuación 4 obteniéndose la distancia de default.

\section{2-El modelo "naive"4}

Desarrollado por Bharath y Shumway (2008) constituye una versión simplificada del modelo formulado por Merton, sin resignar capacidad predictiva conforme surge de la evidencia empírica. En este caso el valor del activo surge de la suma de los valores de mercado del pasivo y patrimonio neto. La pendiente $\mu_{-} \mathrm{A}$, se determina con el rendimiento histórico del activo en el año previo $\left(\mathrm{r}_{\mathrm{e}-(\mathrm{t}-1)}\right)$. La volatilidad del activo está representada por el promedio ponderado entre la volatilidad histórica del patrimonio y del pasivo. La última se supone ad-hoc igual a $\sigma_{\mathrm{D}}=0.05+0.25 \times \sigma_{P N}$. Por lo tanto la volatilidad del activo es $\sigma_{\mathrm{AN}}=[P /(P N+P)] \times \sigma_{D}+[P N /(P N+P)] \times \sigma_{P N}$. La distancia de default para un año es igual a

$$
D D_{N}=\frac{\ln (P N+P) / P+\left(r_{e(t-1)}-\frac{\sigma_{A_{N}}{ }^{2}}{2}\right)}{\sigma_{A_{N}}}(6)
$$

La probabilidad de default es $P D_{N}=N\left(-D D_{N}\right)$.

Una variante de este tipo de modelos es el desarrollado por Afik, Arad y Galil; (2015). En este caso utilizan la volatilidad del patrimonio neto $\left(\sigma_{P N}\right)$ empleando la técnica de RiskMetrics (Longerstaey y Spencer, 1996), a travez de la media móvil exponencial, de la volatilidad recursiva,

$$
\begin{gathered}
\sigma_{t+1}^{2}=\lambda \sigma_{t-1}^{2}+(1-\lambda)_{t}{ }^{2} \text { La pendiente es } \mu_{A}=\max \left(\mathrm{r}_{e(t-1)}, r\right) \text {, siendo DD: } \\
D D_{N S}=\frac{\ln (P N+P) / P+\left(\mu_{A}-\frac{\sigma_{A_{N}}{ }^{2}}{2}\right)}{\sigma_{E}}
\end{gathered}
$$

\section{3-Opciones exóticas barreras:}

Este tipo de modelo reflejan las consecuencias negativas que los incrementos en la volatilidad de los flujos tienen sobre valor del patrimonio. El modelo de opción utilizada es la conocida como barrera del tipo knock out, asimilable a un down and out call. Su valor depende de que el subyacente se encuentre por encima (debajo) del nivel definido como barrera (B), Se agrupan en opciones del tipo knock out option (koo) y knock in options (kio). Las primeras dejan de existir cuando el subyacente alcanza el nivel definido como barrera, las segundas comienzan su existencia alcanzado dicho nivel (Hull, 2005, 2012) ${ }^{6}$. Para estimar probabilidades de fracasos financieros se utilizan del tipo knock out, down and out call, (Brockman y Turtle 2003); (Reiz y Perlich 2007). Adaptando la propuesta de Milanesi, Pesce y El Alabi, (2016), Milanesi (2016), se propone una versión "naive", calculando la volatilidad del activo siguiendo a Bharath y Shumway (2008) e incorporando el cálculo del valor esperado de las probabilidades de default, por mercado, sector o carteras de firmas. El valor de un call tradicional es igual a la suma entras las primas de opciones down and out call (cdo) y down and

\footnotetext{
4 Se denomina "naive" acepción de ingenuo, sencillo o inexperto, producto de su simplicidad.

$5 \lambda$ es una ponderación estimada con metodología RiskMetric (1996), Los extermos se suavizan con desviaciones absolutas $D A_{t}=1 / n \sum_{j=0}^{n-1}\left|r_{j-1}\right|$ y anualizados $\sigma_{M A D}=\sqrt{t \pi / 2} \times M A D, t$ es el total de observaciones anuales.
}

6 Un desarrollo más profundo respecto del funcionamiento de este tipo de opciones se puede encontrar en Milanesi, 2016. 
in call (cdi) (Hull, 2005, 2012); (Haug Gaarder, 2007)7. El valor del patrimonio neto asimilado a una cdo para $B \leq P$, requiere combinar de un call tradicional (c) y un cdi. La ecuación del último es:

$$
\begin{gathered}
c d i=A e^{-q(T-t)}\left(B / A_{0}\right)^{2 \lambda} N(y)-P e^{-r(T-t)}(B / A)^{2 \lambda-2} N\left(y-\sigma_{A} \sqrt{ }(T-t)\right) \\
\lambda=\frac{r-q+\sigma_{A}^{2} / 2}{\sigma_{A}^{2}}(9) \\
y=\frac{\ln \left(B^{2} / A_{o} P\right)}{\sigma_{A} \sqrt{ }(T-t)}+\lambda \sigma_{A} \sqrt{ }(T-t)(10)
\end{gathered}
$$

El $c d o$ se obtiene a partir de la resta entre un call tradicional y un $c d i, c d o=c-c d i$. Para niveles superiores al pasivo $\mathrm{B} \geq \mathrm{P}$; el cdo es igual a,

$$
\begin{gathered}
c d o=A e^{-q(T-t)} N\left(x_{1}\right)-P e^{-r(T-t)} N\left(x_{1}-\sigma_{A} \sqrt{T-t}\right)-A e^{-q(T-t)}\left(B / A_{0}\right)^{2 \lambda} N\left(y_{1}\right)+ \\
P e^{-r(T-t)}(B / A)^{2 \lambda-2} N\left(y_{1}-\sigma_{A} \sqrt{ }(T-t)\right)(11) \\
x_{1}=\frac{\ln \left(V_{0} / B\right)}{\sigma_{A} \sqrt{ }(T-t)}+\lambda \sigma_{A} \sqrt{ }(T-t)(12) \\
y_{1}=\frac{\ln \left(B / V_{o}\right)}{\sigma_{A} \sqrt{ }(T-t)}+\lambda \sigma_{A} \sqrt{ }(T-t(13)
\end{gathered}
$$

Definido un horizonte de duración de la firma, ( $T$ ) las ecuaciones 5, 6 y 7 son empleadas para calcular probabilidad de insolvencia $P\left(A_{T}<P\right)^{8}$ en el caso de $B \leq P$. Asimismo, la probabilidad que el activo adopte valores por debajo de la barrera, previo al vencimiento $\left(t^{*}\right)$ es,

$$
\begin{gathered}
P\left(V_{t *<T}<B\right)=N\left[\frac{\ln (B / A)+\left(R O A-q+\frac{\sigma_{A}^{2}}{2}\right)(T-t)}{\sigma_{A} \sqrt{T-t}}\right]+ \\
\left(B / V_{0}\right)^{\left[2(R O A-q) / \sigma_{A}^{2}\right]-1} N\left[\frac{\ln (B / A)+\left(R O A-q+\frac{\sigma_{A}^{2}}{2}\right)(T-t)}{\sigma_{A} \sqrt{T-t}}\right]
\end{gathered}
$$

La expresión para estimar la probabilidad que en el horizonte T, A sea mayor a B pero inferior a $\mathrm{P}$ es,

$$
\begin{gathered}
P\left(B<A_{T}<P\right)=N\left[\frac{\ln (A / B)+\left(R O A-q+\frac{\sigma_{A}^{2}}{2}\right)(T-t)}{\sigma_{A} \sqrt{T-t}}\right]-N\left[\frac{\ln (A / P)+\left(R O A-q+\frac{\sigma_{A}^{2}}{2}\right)(T-t)}{\sigma_{A} \sqrt{T-t}}\right]- \\
\left(B / V_{0}\right)^{\left[2(R O A-q) / \sigma_{A}^{2}\right]-1} N\left[\frac{\ln (B / A)+\left(R O A-q+\frac{\sigma_{A}^{2}}{2}\right)(T-t)}{\sigma_{A} \sqrt{T-t}}\right]-N\left[\frac{\ln \left(B^{2} / A P\right)+\left(R O A-q+\frac{\sigma_{A}^{2}}{2}\right)(T-t)}{\sigma_{A} \sqrt{T-t}}\right]
\end{gathered}
$$

Finalmente, la probabilidad total de insolvencia es suma de las ecuaciones 14 y 15

$$
\begin{gathered}
P(\text { total })=1-N\left[\frac{\ln (A / P)+\left(R O A-q+\frac{\sigma_{A}^{2}}{2}\right)(T-t)}{\sigma_{A} \sqrt{T-t}}\right]+ \\
\left(B / V_{0}\right)^{\left[2(\mu-q) / \sigma_{A}^{2}\right]-1} N\left[\frac{\ln \left(B^{2} / A \times P\right)+\left(R O A-q+\frac{\sigma_{A}^{2}}{2}\right)(T-t)}{\sigma_{A} \sqrt{T-t}}\right](16)
\end{gathered}
$$

7 Si se suman los perfiles de las opciones barreras de compra del tipo knock out, (in-out) se observa que cuando una expira la otra se activa recíprocamente, por lo tanto el resultado es del de un call regular.

8 Cuando $\mathrm{B} \geq \mathrm{P}$, las probabilidades de insolvencia al vencimiento son explicadas de manera similar a los modelos tradicionales. De verificarse la insolvencia si B es > P (deuda) entonces el activo (A) debe ser menor al pasivo. En este escenario el subyacente estará por debajo de B e inclusive de P. Un cdo deja de existir cuando el subyacente está por debajo de B; (Reiz y Perlich; 2007) 


\section{4-El valor esperado de default del mercado de capitales.}

Las ecuaciones 6 y 16 son la base para estimar el valor probable de default de un mercado,

$$
\begin{gathered}
V E_{\text {naive }}(\text { default })=\sum_{i=1}^{n}(C B+P)_{t, i} \times P(D D)_{t, i}(17) \\
V E_{c d i}(\text { default })=\sum_{i=1}^{n}(C B+P)_{t, i} \times P\left(D_{c d i}\right)_{t, i}(18)
\end{gathered}
$$

Este surge sumar CB (capitalización bursátil) y P (pasivo) por cada empresa integrante de la cartera multiplicada por la probabilidad e default para los modelos naive y opciones exóticas ${ }^{9}$.

\section{3- El modelo naive versus opciones barrera. Análisis de caso.}

En la presente sección se ilustrará el funcionamiento del modelo empleando un caso hipotético. Para ello se supone un activo es $A=\$ 200$; pasivo ${ }^{10} P=\$ 125$ con vencimiento $T=10$ años; tasa libre de riesgo $r=5 \%$ anual, volatilidad ${ }^{11}$ del activo y del patrimonio $\sigma_{A}=30 \%$ y $\sigma_{E}=72 \%$. El ratio de pago de dividendos (q) $3 \%$ y la tasa de rendimiento de los activos (ROA) del 10\%. Para el modelo naive se utilizan la ecuación 6 (naive) y las expresiones 8 a 16 para modelo de opciones barreras.

El valor del patrimonio en el modelo naive es: $P N=A e^{-q(T-t)} N\left(d_{1}\right)-P e^{-r(T-t)} N\left(d_{2}\right)$, $d_{1}$ 1.7076; $d_{2}$ 0,7589; $N\left(d_{1}\right)$ 0,95614; $N(d 2)$ 0,77605, siendo su valor de $\$ 105,97$. Seguidamente es presentado el análisis de sensibilidad bivariado ${ }^{12}$ que relaciona valor del patrimonio, con volatilidad (columna) y endeudamiento (fila).

Tabla 1: Relación volatilidad-endeudamiento y valor del patrimonio neto según el modelo BS

\begin{tabular}{|l|llllllllll|}
\hline$\sigma / P$ & $\$ 50,00$ & $\$ 80,00$ & $\$ 100,00$ & $\$ 115,00$ & $\$ 125,00$ & $\$ 150,00$ & $\$ 180,00$ & $\$ 200,00$ & $\$ 205,00$ & $\$ 210,00$ \\
\hline $10 \%$ & $\$ 129,77$ & $\$ 118,73$ & $\$ 111,38$ & $\$ 105,86$ & $\$ 102,18$ & $\$ 92,99$ & $\$ 82,00$ & $\$ 74,74$ & $\$ 72,94$ & $\$ 71,15$ \\
$30 \%$ & $\$ 129,99$ & $\$ 119,78$ & $\$ 113,41$ & $\$ 108,88$ & $\$ 105,98$ & $\$ 99,13$ & $\$ 91,63$ & $\$ 87,03$ & $\$ 85,93$ & $\$ 84,84$ \\
$60 \%$ & $\$ 134,86$ & $\$ 129,27$ & $\$ 126,05$ & $\$ 123,84$ & $\$ 122,45$ & $\$ 119,22$ & $\$ 115,72$ & $\$ 113,59$ & $\$ 113,07$ & $\$ 112,57$ \\
$90 \%$ & $\$ 141,35$ & $\$ 138,94$ & $\$ 137,58$ & $\$ 136,65$ & $\$ 136,06$ & $\$ 134,70$ & $\$ 133,23$ & $\$ 132,32$ & $\$ 132,10$ & $\$ 131,88$ \\
$120 \%$ & $\$ 145,46$ & $\$ 144,59$ & $\$ 144,10$ & $\$ 143,77$ & $\$ 143,56$ & $\$ 143,07$ & $\$ 142,53$ & $\$ 142,21$ & $\$ 142,13$ & $\$ 142,05$ \\
\hline
\end{tabular}

(elaboración propia)

Frente a incrementos en el endeudamiento (ejercicio), el efecto es la disminución en el valor del patrimonio (prima). Los aumentos de riesgo (volatilidad) incrementan el valor del patrimonio. Similares conclusiones respecto de la sensibilidad sobre el horizonte temporal, en efecto para $T$ - $t$, en $T-9=1 P N$ : $\$ 81,61 ; T-5=5 ; P N: \$ 100,27 \mathrm{y}$ $T-0=10 ; P N: \$ 105,98$.

9 Las ecuaciones 17 y 18 suponen no correlación de probabilidad de insolvencia entre empresas. 10 El pasivo total de la firma se asimila a un bono cupón cero, expresándose por su valor nominal. 11 Se supone una volatilidad del activo que surge de aplicar la ecuación desapalancamiento sobre la volatilidad del patrimonio neto $\sigma_{A}=\sigma_{E} /(1+(\mathrm{B} /(\mathrm{S})(1-\mathrm{t}))$.

12 La sensibilidad se realizó con la herramientas tablas del aplicativo Microsoft Excel ${ }^{\circledR}$. 
Para una opción knock out $\mathrm{B}<\mathrm{P}$, se supone una barrera del $92 \%$ del valor del pasivo. Aplicando las ecuaciones 8, 9 y 10 se tiene: $\lambda=1,2777 ; y=0,54099$ y $N(y)=0,735288$. El $c d i$ asciende a $\$ 13,82$, y el $c d o=c$ - $c d i$; siendo de $\$ 92,1587=(\$ 105,97-\$ 13,82)$. Ilustrativamente se calcula el valor para $B>P$ (ecuaciones 11,12 y 13), donde: $\mathrm{x}_{1}=1$, $7955 ; y_{1}=0,6288 ; \mathrm{N}\left(x_{1}\right)=0,963715 ; N\left(y_{1}\right)=0,73528$. El valor se integra por valor del call (primer término) $(\$ 105,93)$ y cdi $(\$ 13,82)$ (segundo término), obteniendo el mismo resultado que la ecuación 8 . A continuación se presentan los valores correspondientes al patrimonio, frente a diferentes niveles de volatilidad (columna) y barreras (fila) ${ }^{13}$.

Tabla 2: Relación volatilidad-barrera y valor del patrimonio neto según opción exótica knock out (elaboración propia)

\begin{tabular}{|l|cccccccccc|}
\hline$\sigma / P$ & $\$ 50,00$ & $\$ 80,00$ & $\$ 100,00$ & $\$ 115,00$ & $\$ 125,00$ & $\$ 150,00$ & $\$ 180,00$ & $\$ 200,00$ & $\$ 205,00$ & $\$ 210,00$ \\
\hline $10 \%$ & $\$ 102,18$ & $\$ 102,18$ & $\$ 102,18$ & $\$ 102,17$ & $\$ 102,11$ & $\$ 101,30$ & $\$ 83,50$ & $\$ 0,40$ & $\$-49,04$ & $\$-119,18$ \\
$30 \%$ & $\$ 106,72$ & $\$ 104,14$ & $\$ 98,74$ & $\$ \mathbf{9 2 , 1 5 9}$ & $\$ 83,11$ & $\$ 66,83$ & $\$ 32,43$ & $\$ 2,29$ & $\$-8,77$ & $\$-17,95$ \\
$60 \%$ & $\$ 117,06$ & $\$ 100,92$ & $\$ 87,62$ & $\$ 76,59$ & $\$ 64,77$ & $\$ 47,92$ & $\$ 20,58$ & $\$ 1,11$ & $\$-5,13$ & $\$-10,33$ \\
$90 \%$ & $\$ 117,33$ & $\$ 96,55$ & $\$ 81,75$ & $\$ 70,26$ & $\$ 58,48$ & $\$ 42,38$ & $\$ 17,48$ & $\$ 0,45$ & $\$-4,36$ & $\$-8,74$ \\
$120 \%$ & $\$ 115,61$ & $\$ 93,79$ & $\$ 78,79$ & $\$ 67,35$ & $\$ 55,76$ & $\$ 40,12$ & $\$ 16,27$ & $\$ 0,16$ & $\$-4,07$ & $\$-8,15$ \\
\hline
\end{tabular}

(elaboración propia)

Conforme surge de la tabla, más cercana el valor de la barrera en relación al valor de los activos menor es el valor del patrimonio de la firma, producto de mayores probabilidades de insolvencia. Respecto del horizonte temporal, no existe una relación directa valor distancia temporal, en especial para valores de activos cercanos a niveles de barrera.

Tabla 3: Relación horizonte temporal-barrera y valor del patrimonio neto según opción exótica knock out (elaboración propia).

\begin{tabular}{|l|lllll|}
\hline $\mathbf{( T - t )} / \mathbf{P}$ & $\mathbf{\$ 8 0 , 0 0}$ & $\mathbf{\$ 1 0 0 , 0 0}$ & $\mathbf{\$ 1 1 5 , 0 0}$ & $\mathbf{\$ 1 3 0 , 0 0}$ & $\mathbf{\$ 1 5 0 , 0 0}$ \\
\hline (T-t)=10 & $\$ 81,61$ & $\$ 81,61$ & $\$ 81,61$ & $\$ 81,61$ & $\$ 81,61$ \\
$(\mathrm{~T}-\mathrm{t})=5$ & $\$ 100,28$ & $\$ 100,28$ & $\mathbf{\$ 1 0 0 , 2 8}$ & $\mathbf{\$ 1 0 0 , 2 8}$ & $\mathbf{\$ 1 0 0 , 2 8}$ \\
$(\mathrm{T}-\mathrm{t})=1$ & $\$ 105,98$ & $\$ 105,98$ & $\$ 105,98$ & $\mathbf{\$ 1 0 5 , 9 8}$ & $\$ 105,98$ \\
\hline
\end{tabular}

(elaboración propia)

Seguidamente son estimadas las probabilidades de default para el modelo naive (ecuaciones 5 y 6 ).

13 Para niveles $\mathrm{B} \leq \mathrm{P}$ se aplicó la ecuación 5 mientras que en el caso de B>P se utilizó la ecuación 8 . 
Tabla 4: Relación volatilidad-endeudamiento y probabilidad de insolvencia según opción convencional para un horizonte $\mathrm{t}=10$.

\begin{tabular}{|l|ccccc|}
\hline $\boldsymbol{\sigma} / \mathbf{P}$ & $\mathbf{\$ 8 0 , 0 0}$ & $\mathbf{\$ 1 0 0 , 0 0}$ & $\mathbf{\$ 1 1 5 , 0 0}$ & $\mathbf{\$ 1 2 5 , 0 0}$ & $\mathbf{\$ 1 5 0 , 0 0}$ \\
\hline $10 \%$ & $0,00 \%$ & $0,00 \%$ & $0,00 \%$ & $0,00 \%$ & $0,00 \%$ \\
$30 \%$ & $2,06 \%$ & $6,11 \%$ & $9,50 \%$ & $\mathbf{1 4 , 1 1 \%}$ & $15,06 \%$ \\
$70 \%$ & $51,15 \%$ & $59,53 \%$ & $63,38 \%$ & $67,10 \%$ & $67,74 \%$ \\
\hline
\end{tabular}

(elaboración propia)

Las probabilidades de insolvencia obtenidas del modelo de opciones barreras naive requiere de aplicar las ecuaciones 14,15 y $16^{14}$. Las probabilidades son: $P\left(A_{t^{*}<T}<B\right) 34,45 \%, P\left(B<A_{T}<P\right) \quad 13,07 \%$, probabilidad total $P($ total $)=34,45 \%+13,07 \%=$ $47,52 \%$. La tabla presenta la sensibilidad entre probabilidad de default para niveles de endeudamiento y volatilidad.

Tabla 5: Relación volatilidad-endeudamiento y probabilidad de insolvencia según opción exótica del tipo knock out, para un horizonte $\mathrm{t}=10$

\begin{tabular}{|l|cccccccc|}
\hline O/A (T total) & $\mathbf{\$ 5 0 , 0 0}$ & $\mathbf{\$ 8 0 , 0 0}$ & $\mathbf{\$ 1 0 0 , 0 0}$ & $\mathbf{\$ 1 1 5 , 0 0}$ & $\mathbf{\$ 1 2 5 , 0 0}$ & $\mathbf{\$ 1 5 0 , 0 0}$ & $\mathbf{\$ 1 8 0 , 0 0}$ & $\mathbf{\$ 2 0 0 , 0 0}$ \\
\hline $10 \%$ & $0,02 \%$ & $0,02 \%$ & $0,02 \%$ & $0,06 \%$ & $0,18 \%$ & $2,29 \%$ & $25,39 \%$ & $100,00 \%$ \\
$30 \%$ & $23,10 \%$ & $29,63 \%$ & $38,81 \%$ & $\mathbf{4 7 , 5 2 \%}$ & $53,84 \%$ & $70,17 \%$ & $88,82 \%$ & $100,00 \%$ \\
$70 \%$ & $80,88 \%$ & $87,21 \%$ & $90,58 \%$ & $92,70 \%$ & $93,94 \%$ & $96,53 \%$ & $98,84 \%$ & $100,00 \%$ \\
\hline
\end{tabular}

(elaboración propia)

La tabla 5 presenta un análisis de sensibilidad donde se relaciona diferentes niveles de barrera con diferentes niveles de volatilidad, a los efectos de estudiar el comportamiento de las probabilidades de insolvencia. El modelo captura la relación positiva bivariada entre el riesgo en los flujos de fondos (aumento de volatilidad), aumentos del endeudamiento y mayor probabilidad de insolvencia, presentando un mayor ajuste que el modelo tradicional. Para un nivel del 30\% de volatilidad y un rango de deuda de $\$ 80$ a $\$ 150$ las probabilidades de default oscilan del $2 \%$ al $15 \%$. En el modelo de opciones barreras estas van desde el 29\% al 70\%.

Tabla 6: Relación horizonte temporal-endeudamiento y probabilidad de insolvencia según opción exótica del tipo knock out para $\sigma=30 \%$

\begin{tabular}{|c|c|c|c|c|c|c|c|c|}
\hline o/A (T total) & $\$ 50,00$ & $\$ 80,00$ & $\$ 100,00$ & $\$ 115,00$ & $\$ 125,00$ & $\$ 150,00$ & $\$ 180,00$ & $\$ 200,00$ \\
\hline$(\mathrm{T}-\mathrm{t})=1$ & $4,95 \%$ & $4,95 \%$ & $5,05 \%$ & $6,47 \%$ & $10,26 \%$ & $38,56 \%$ & $83,07 \%$ & $100,00 \%$ \\
\hline$(\mathrm{T}-\mathrm{t})=5$ & $18,78 \%$ & $20,71 \%$ & $26,86 \%$ & $35,13 \%$ & $42,13 \%$ & $62,36 \%$ & $86,34 \%$ & $100,00 \%$ \\
\hline$(\mathrm{T}-\mathrm{t})=10$ & $23,10 \%$ & $29,63 \%$ & $38,81 \%$ & $47,52 \%$ & $53,84 \%$ & $70,17 \%$ & $88,82 \%$ & $100,00 \%$ \\
\hline
\end{tabular}

(elaboración propia)

14 En el caso donde la barrera sea mayor al activo B>P , la ecuación no tiene sentido de ser aplicada, puesto que la firma no ingresa en insolvencia si los activos se encuentre por encima del nivel de barrera. 
La tabla 6 presenta la relación positiva entre tiempo, deuda y probabilidades de default. A mayor horizonte temporal, la volatilidad crece replicando la mayor incertidumbre en los negocios futuros.

\section{4- Probabilidades de default en empresas cotizantes del mercado de capitales argentinos.}

Son aplicados los modelos analizados en el punto anterior sobre dos firmas con diferente salud financiera y, seguidamente sobre un conjunto de firmas cotizantes en el mercado de capitales de Argentina. En el primer caso se calculó el valor del patrimonio neto como opción de compra y las probabilidades de default ex post, durante la serie de temporal seleccionada, con el fin de analizar la coherencia en el comportamiento de los modelos. Fueron seleccionadas dos firmas, una con dificultades financieras y la otra con una situación financiera estable. Se estudió la firma Pertrak S.A (sector industria y manufacturas) durante el periodo 2009 al 2014, a partir de dicho momento comenzó su proceso de reestructuración, reorganización empresarial y eliminación del listado de cotizantes en el mercado de capitales, producto de sus dificultades financieras. Los resultados son comparados con los obtenidos por la firma Pampa Energía S.A (sector energético).

Seguidamente se realiza un estudio descriptivo consistente en calcular por sector y globalmente las probabilidades y valor esperado de default, (ecuaciones 17 y 18). En este caso la muestra se compone de 53 empresas clasificadas por sector ${ }^{15}$, siendo el punto de observación la información financiera disponible a diciembre del 2018.

En ambos casos, los valores correspondientes al activo (A), pasivo (P), resultado operativo (RO) y patrimonio neto (PN) fueron obtenidos de los reportes contables a fecha de las observaciones publicados por la Bolsa de Comercio de Buenos Aires y la Comisión Nacional de Valores ${ }^{16}$ (anexo, tabla 1.A). Los valores correspondientes a volatilidad del activo, patrimonio neto y pasivo fueron calculados empleando el modelo de Bharath y Shumway, (2008). En el caso de la volatilidad del patrimonio $\left(\sigma_{P N}\right)$, surge del valor publicado por Instituto Argentino de Mercados de Capitales. La volatilidad del pasivo se obtuvo con la expresión $\sigma_{D}=0.05+0.25 \times \sigma_{P_{N}}$. Finalmente, la volatilidad del activo es $\sigma_{A N}=[\mathrm{P} /(\mathrm{PN}+\mathrm{P})] \times \sigma_{D}+[\mathrm{PN}(\mathrm{PN}+\mathrm{P})] \times \sigma_{P N}$. El (ROA) de cada firma es el cociente entre resultado operativo y activo contable. La tasa libre de riesgo ( $\mathrm{r}$ ) es la Baibar publicada por el IAMC, correspondiente a cada observación para la serie de tiempo y a diciembre del 2018. En cada caso se dispone un horizonte $T=10$ años y un nivel de barrera de $90 \%$ del pasivo contable $(B<P)$. Las tablas 2.A a 5.A del anexo exponen por unidad de análisis las variables de entrada de los modelos, parámetros del modelo naive y valor del patrimonio como opción, parámetros y valores del patrimonio como cdo directo y por diferencia, finalmente las probabilidades y valor de default.

\section{1-Análisis comparativo de series de tiempo empresas con y sin dificultades financieras}

La siguiente tabla presenta la serie de tiempo de probabilidades de default correspondiente a la firma Pertrak. S.A, a los efectos de realizar un estudio ex - post de la capacidad predictiva relativa a los modelos estudiados. El poder predictivo del

15 Es respetada la clasificación sectorial elaborada por Bolsa de Comercio de Buenos Aires (BCBA) www. bolsar.com e Instituto Argentino de Mercado de Capitales (IAMC) www.iamc.com.ar

16 www.bolsar.com.ar y www.cnv.gov.ar 
modelo naive es satisfactorio, reforzando los resultados obtenidos en las pruebas empíricas (Bharath y Shumway, 2008), No obstante el modelo de opciones barreras (CDO) presenta valores levemente superiores en relación a las probabilidad de fracasos financieros. Esto es producto de su estructura conforme fue expuesto en el apartado precedente, ya que los incrementos de volatilidad no generen sesgos positivos en el valor del patrimonio de la firma y por ende en las probabilidades de que el subyacente supere el precio ejercicio. En tal sentido los valores del patrimonio obtenidos siguen la misma lógica.

Tabla 7: Variables, probabilidades de default y valor esperado de default Pertrak S.A 2014-2009

\begin{tabular}{|c|c|c|c|c|c|c|}
\hline Conceptos & 2014 & 2013 & 2012 & 2011 & 2010 & 2009 \\
\hline Activo contable & 50,7 & 49,4 & 40,3 & 50,5 & 42,6 & 42,1 \\
\hline Deuda & 37,5 & 38,1 & 37,9 & 38,3 & 31,6 & 29,5 \\
\hline PN & 13,2 & 11,4 & 2,4 & 12,2 & 10,9 & 12,6 \\
\hline ROA & $-6,83 \%$ & $-8,60 \%$ & $-14,54 \%$ & $-3,49 \%$ & $-14,02 \%$ & $-15,11 \%$ \\
\hline$\sigma e$ & $41,00 \%$ & $39,45 \%$ & $38,77 \%$ & $37,22 \%$ & $36,77 \%$ & $39,29 \%$ \\
\hline$\sigma \mathrm{d}$ & $15,25 \%$ & $14,86 \%$ & $14,69 \%$ & $14,31 \%$ & $14,19 \%$ & $14,82 \%$ \\
\hline$\sigma \mathrm{a}$ & $21,94 \%$ & $20,51 \%$ & $16,12 \%$ & $19,85 \%$ & $19,98 \%$ & $22,17 \%$ \\
\hline Baibar & $26,60 \%$ & $23,30 \%$ & $10,83 \%$ & $10,13 \%$ & $9,62 \%$ & $10,00 \%$ \\
\hline Prob default naive & $81,54 \%$ & $89,38 \%$ & $99,86 \%$ & $66,62 \%$ & $98,06 \%$ & $97,71 \%$ \\
\hline Valor naive & 48,06 & 45,73 & 27,52 & 36,69 & 30,58 & 31,41 \\
\hline Prob default CDO & $90,70 \%$ & $95,08 \%$ & 99,96\% & $80,97 \%$ & $99,12 \%$ & $98.95 \%$ \\
\hline Valor CDO & 47,73 & 45,23 & 22,72 & 34,47 & 28,81 & 29,64 \\
\hline Valor Mercado & 18,48 & 17,82 & 13,69 & 25,43 & 16,74 & 16,74 \\
\hline $\mathrm{P} / \mathrm{A}$ & 0,74 & 0,77 & 0,94 & 0,76 & 0,74 & 0,70 \\
\hline Valor naive/VM & 2,60 & 2,57 & 2,01 & 1,44 & 1,83 & 1,88 \\
\hline Valor CDO/VM & 2,58 & 2,54 & 1,66 & 1,36 & 1,72 & 1,77 \\
\hline
\end{tabular}

(elaboración propia)

La segunda tabla aplica el modelo sobre la firma Pampa Energía S.A, una firma con situación financiera estable. En los últimos tres años experimento un incremento en su apalancamiento financiero, ya que la razón deuda-activos se incrementó desde el 12\% al 70\%. En tal sentido, el modelo CDO demuestra ser más sensible a tales situaciones. Sin perjuicio que los conductores de valor resumidos en el ROA representan una mejoría, las probabilidades de default se ajustan al alza (25\%) en relación al modelo naive. El modelo CDO morigera los efectos positivos de incrementos en la volatilidad frente a incrementos del endeudamiento (precio de ejercicio). 
Tabla 8: Variables, probabilidades de default y valor esperado de default Pampa Energía S.A 2018-2009

\begin{tabular}{|c|c|c|c|c|c|c|c|c|c|c|}
\hline Conceptos & 2018 & 2017 & 2016 & 2015 & 2014 & 2013 & 2012 & 2011 & 2010 & 2009 \\
\hline Activo & 147128 & 73472 & 47297 & 7933 & 3743 & 2603 & 2136 & 2720 & 3523 & 3601 \\
\hline Deuda & 95605 & 56421 & 34891 & 942 & 822 & 504 & 335 & 362 & 242 & 264 \\
\hline PN & 51523 & 17051 & 12406 & 6991 & 2920 & 2099 & 1801 & 2359 & 3281 & 3337 \\
\hline ROA & $16,91 \%$ & $8,28 \%$ & $4,29 \%$ & $42,16 \%$ & $24,22 \%$ & $15,54 \%$ & $0,39 \%$ & $-1,56 \%$ & $-1,23 \%$ & $6,55 \%$ \\
\hline$\sigma e$ & $55,29 \%$ & $31,01 \%$ & $37,37 \%$ & $67,38 \%$ & $64,90 \%$ & $69,84 \%$ & $39,38 \%$ & $31,67 \%$ & $46,09 \%$ & $26,36 \%$ \\
\hline$\sigma d$ & $18,82 \%$ & $12,75 \%$ & $14,34 \%$ & $21,85 \%$ & $21,23 \%$ & $22,46 \%$ & $14,85 \%$ & $12,92 \%$ & $16,52 \%$ & $11,59 \%$ \\
\hline$\sigma a$ & $31,59 \%$ & $16,99 \%$ & $20,38 \%$ & $61,97 \%$ & $55,30 \%$ & $60,66 \%$ & $35,53 \%$ & $29,18 \%$ & $44,06 \%$ & $25,28 \%$ \\
\hline Baibar & $42,67 \%$ & $25,50 \%$ & $25,61 \%$ & $21,59 \%$ & $26,60 \%$ & $23,30 \%$ & $10,83 \%$ & $10,13 \%$ & $9,62 \%$ & $10,00 \%$ \\
\hline $\begin{array}{l}\text { Prob } \\
\text { default } \\
\text { naive }\end{array}$ & $5,21 \%$ & $3,89 \%$ & $20,73 \%$ & $1,20 \%$ & $8,42 \%$ & $23,99 \%$ & $13,11 \%$ & $5,98 \%$ & $12,77 \%$ & $0,01 \%$ \\
\hline $\begin{array}{l}\text { Valor } \\
\text { naive }\end{array}$ & 145787 & 69067 & 44602 & 7831 & 3687 & 2557 & 2023 & 2589 & 3431 & 3504 \\
\hline $\begin{array}{l}\text { Prob } \\
\text { default } \\
\text { CDO }\end{array}$ & $24,90 \%$ & $15,21 \%$ & $41,69 \%$ & $4,58 \%$ & $20,72 \%$ & $41,37 \%$ & $18,81 \%$ & $8,10 \%$ & $17,67 \%$ & $0,02 \%$ \\
\hline $\begin{array}{l}\text { Valor } \\
\text { CDO }\end{array}$ & 144934 & 69002 & 44427 & 7786 & 3653 & 2521 & 2021 & 2589 & 3430 & 3504 \\
\hline $\begin{array}{l}\text { Valor } \\
\text { Mercado }\end{array}$ & 88819 & 91641 & 40403 & 19672 & 5796 & 2484 & 1275 & 2576 & 3628 & 2747 \\
\hline $\mathrm{P} / \mathrm{A}$ & 0,65 & 0,77 & 0,74 & 0,12 & 0,22 & 0,19 & 0,16 & 0,13 & 0,07 & 0,07 \\
\hline $\begin{array}{l}\text { Valor } \\
\text { naive/VM }\end{array}$ & 1,64 & 0,75 & 1,10 & 0,40 & 0,64 & 1,03 & 1,59 & 1,01 & 0,95 & 1,28 \\
\hline $\begin{array}{l}\text { Valor } \\
\mathrm{CDO} / \mathrm{VM}\end{array}$ & 1,63 & 0,75 & 1,10 & 0,40 & 0,63 & 1,01 & 1,59 & 1,01 & 0,95 & 1,28 \\
\hline
\end{tabular}

(elaboración propia)

\section{2-Análisis de las probabilidades y valor esperado de default en el mercado argentino}

Seguidamente se exponen los resultados agrupados por sectores económicos. En este caso el modelo se aplicó con fines descriptivos, en un primer nivel, de la situación financiera por sector. Los resultados obtenidos son de carácter preliminar, ya que los guarismos expuestos deben analizarse en relación a los conductores de valor y fundamentals de las firmas que integran cada sector, no siendo el objetivo del presente trabajo. Las tablas contienen los valores correspondientes a probabilidades de default promedio, valor esperado de default agregado y la proporción del valor esperado de default sobre el valor total. 


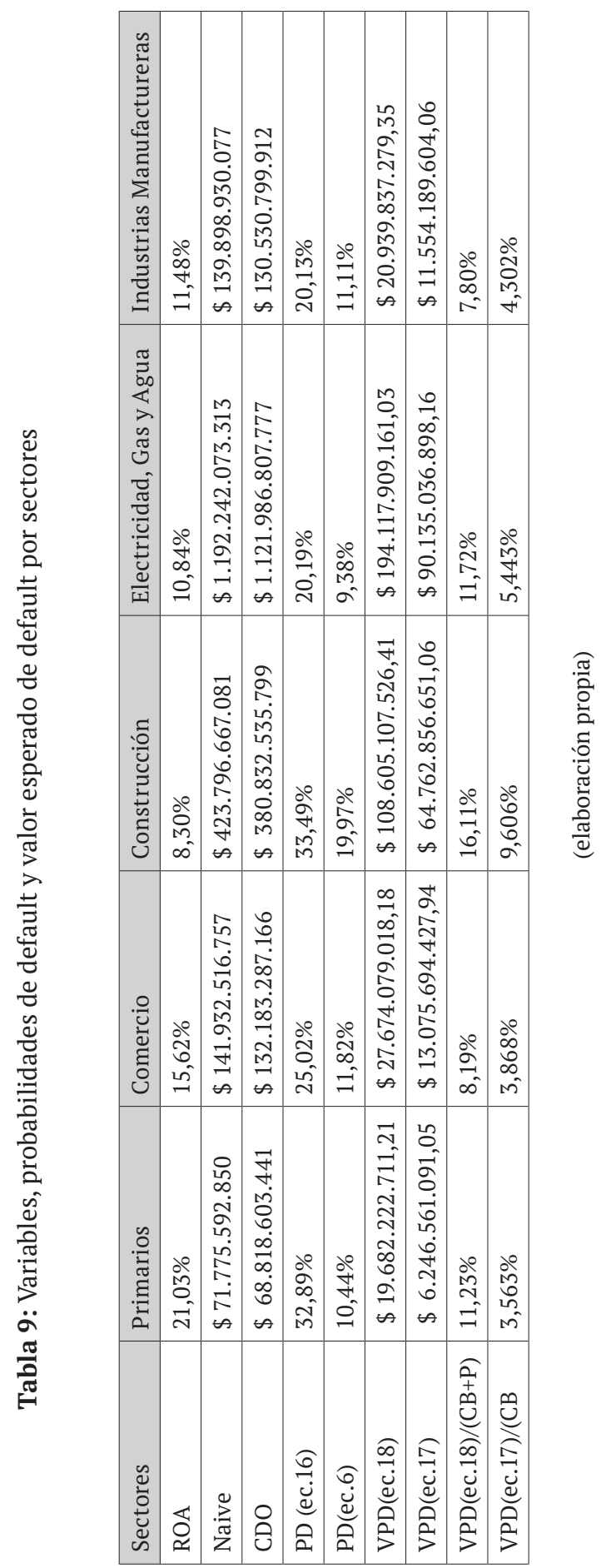




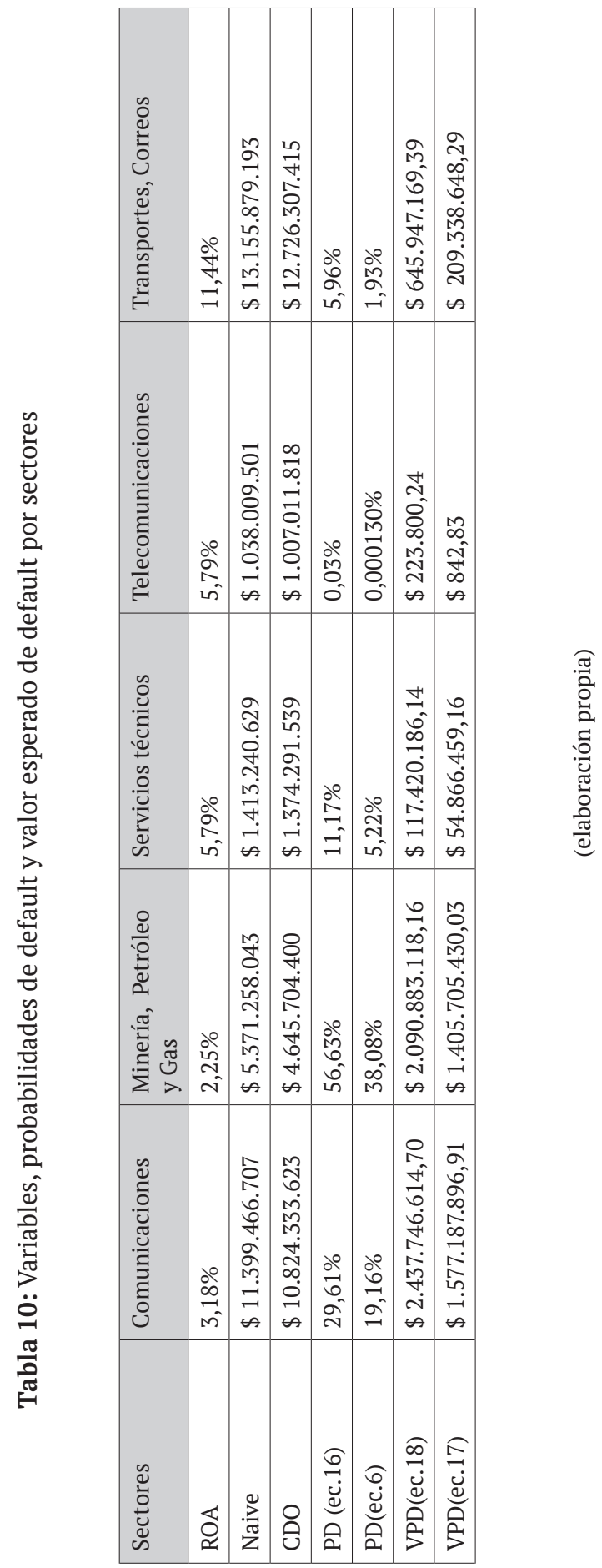


Los datos agregados para el mercado argentino son expuestos en la siguiente tabla

Tabla 11: Variables y probabilidades de default mercado argentino. Diciembre 2018.

\begin{tabular}{|l|l|}
\hline Variables & Total Mercado \\
\hline Activo & $\$ 1.605 .430 .171 .880$ \\
\hline ROA & $11,5185 \%$ \\
\hline Naive & $\$ 1.999 .413 .280 .395$ \\
\hline CDO & $\$ 1.810 .354 .365 .663$ \\
\hline PD (ec.16) & $24,99 \%$ \\
\hline PD(ec.6) & $12,99 \%$ \\
\hline CB & $\$ 3.041 .182 .783 .880,33$ \\
\hline VPD(ec.18) & $\$ 401.146 .656 .319,77$ \\
\hline VPD(ec.17) & $\$ 208.586 .109 .896,31$ \\
\hline VPD(ec.18)/CB & $13,19 \%$ \\
\hline VPD(ec.17)/CB & $6,859 \%$ \\
\hline
\end{tabular}

(elaboración propia)

Se calculó la correlación entre datos transversales de volatilidad del activo por firma (tabla 2.A) y probabilidades de default los modelos (tabla 5.A). Se observa una mayor correlación positiva entre el corte transversal de firmas para el periodo 2018 entre volatilidad y probabilidad de default en el modelo de opciones barrera.

Tabla 12: Correlaciones volatilidad activo probabilidad insolvencia (elaboración propia)

\begin{tabular}{|l|l|}
\hline Parámetros & Coeficiente Correlación \\
\hline oa/PD (ec.16) & 0,28167513 \\
\hline oa/PD (ec.6) & 0,17797248 \\
\hline
\end{tabular}

(elaboración propia)

En relación a las probabilidades de default, en promedio se evidencia un importante porcentaje que asciende al 25\%. En términos de valor, el impacto representa un $13 \%$ del valor de mercado de las firmas involucradas. En principio, y relacionado con el periodo analizado, los resultados son coherentes con la situación macro económica del mercado objetivo. Paralelamente, ponen de manifiesto el carácter dinámico del modelo, ya que el valor de la firma se relaciona directamente con el valor del activo, suponiendo que sigue un proceso estocástico geométrico browniano. Este movimiento es comandado por el rendimiento operativo (ROA) y la volatilidad del activo. En el caso de las opciones barreras, los excesivos niveles de volatilidad impactan negativamente 
sobre el valor del patrimonio, ajustándose a la dinámica empresarial. En esa línea de razonamiento se encuentran los resultados obtenidos a través de ratios financieros y regresiones logit sobre paneles de datos, en firmas Argentinas en la década del 1990, (Sandin y Porporato M, 2007); (Caro, Diaz y Porporato, 2013). En especial las razones de rentabilidad, endeudamiento (solvencia) y flujos de fondos son las que presentan significatividad estadística para explicar probabilidad de fracasos financieros ${ }^{17}$.

\section{5-Conclusiones}

Los resultados obtenidos a partir del análisis de casos, el estudio comparativo entre dos firmas y la aplicación de los modelos en firmas cotizantes para el mercado seleccionado, pusieron de manifiesto las ventajas del modelo de opciones barreras naive, en relación a su par basado en la lógica de opciones simples. En primer lugar, las opciones barreras presentan una relación positiva entre el valor del patrimonio y los niveles de volatilidad, hasta determinando punto en donde excesivos niveles de riesgo impactan negativamente en el valor de la empresa. En segundo lugar, la muestra arrojó un coeficiente de correlación mayor entre las probabilidades de default y riesgo operativo. Los resultados obtenidos son coherentes con aquellos provenientes de trabajos donde fueron empleados ratios financieros y modelos de regresión logit con datos de panel, ya que las razones financieras con mayor significatividad estadística para predecir fracasos fueron los ratios de rentabilidad, solvencia y flujos de fondos. Se encuentran contenidos en las estructuras correspondientes a las ecuaciones que describen los modelos estudiados en el presente trabajo. El valor de la firma es función de la evolución del activo, explicada por el comportamiento estocástico del rendimiento operativo y su volatilidad. El pasivo (solvencia) se constituye en la barrera o precio de ejercicio dentro de la lógica contenida en los modelos de opciones. En tal sentido, las opciones barreras son una útil herramienta de predicción de probabilidades y valor de default. Respetan la lógica de la elección de inversiones y dinámica de negocios, donde mayores niveles de riesgo no necesariamente se traducen en mayor valor del patrimonio. En tal sentido, un instrumento aplicable a nivel de empresa, sector o agregado económico, para el empresario como para los formadores de políticas públicas.

\section{Declaración de conflicto de interés}

Los autores del presente manuscrito manifiestan que no existen conflictos de interés con ninguna entidad o institución, ni de carácter personal en esta publicación.

\footnotetext{
17 Otras investigaciones encontraron poca evidencia estadística a partir de aplicar el modelo Z-Score y la relaciones entre liquidez, rentabilidad y riesgo de quiebra con la probabilidades de fracasos financieros (Andrade-Valbuena, Moscoso Duran, Salcedo-Perez, 2017). En este caso, se deba a que la prueba econométrica se llevó a cabo con el coeficiente de correlación de Pearson, en lugar de utilizar datos de panel con modelos de regresión logit
} 


\section{Referencias}

Afik, Z, Arada, O y Galil, K. (2015). Using Merton modelo for default prediction: An empirical assessment of selected alternatives. Journal of Empirical Finance, 35, 4367.

Altman, E. (1968). Financial ratios, discriminant analysis and the predicition of corporate bankruptcy. Journal of Finance, 4, 589-609.

Altmant, E. (1993). Corporate Financial Distress and Bankrutpcy (Second ed.). New York, United State: Wiley Finance.

Altmant, E y Kishore, M. (1996). Almost everything you wanted to know about recoveries on default bonds. Financial Analyst Journal(52), 57-64.

Andrade-Valbuena, N, Moscoso Duran F y Salcedo-Perez, C. (2017). Liquidity, Profitability, Tax rate and Bankruptcy risk in Colombian SMEs. Estudios de Administración, 24(1), 16-32.

Bharath, S y Shumway, T. (2008). Forescasting Default with the Merton distance to default model. Review of Financial Studies, 23, 589-609.

Black, F y Scholes, M. (1973). The pricing of options and corporate liabilities. Journal of Political Economics(81), 637-659.

Brockman, P y Turtle, H. (2003). A Barrier Option Framework for Corporate Security Valuation. Journal of Financial Economics, 67, 511-529.

Caro, N, Diaz, M y Porporato, M. (2013). Predicción de quiebras empresariales en economías emergentes: Uso de un modelo logístico mixto. Revista de Métodos Cuantitativos para la Economía y Empresa, 16, 200-2015.

Castro Monge, E. (2010). El estudio de casos como metodología de investigación y su importancia en la dirección y administración de empresas. Revista Nacional de Administración, 2(1), 31-54.

Cochrane, J. (2005). Asset Pricing (2 ed.). Princeton: Princeton University Press.

Copeland, T y Antikarov, V. (2001). Real Options (1 ed.). New York: Texere LLC.

Crosbie, P y Bohn J. (2002). Modeling defaukl risk. San Francisco, United State: KMV Corporation.

Crosbie, P y Bohn, J. (2003). Modeling Default Risk. WP Moody's KMV, http://www. business.illinois.edu/gpennacc/moodyskmv.pdf.

Dixit, A y Pindyck, R. (1994). Investment under Uncertainty (1 ed.). New Jersey: Pricenton University Press. 
Graziabile, D. (2012). Derecho Concursal. CABA, Argentina: Abeledo Perrot.

Haug Gaarder, E. (2007). Derivatives: Models ond Models (1 ed.). Chichester : John Wiley \& Sons.

Hillegeist, S, Keating, E, Cram, D y Lundstedt, K. (2004). Assesing the probability of bankruptcy. Review of Accounting Studies(9), 5-34.

Hull, J. (2005). Futures, Options and other Derivatives (5 ed.). New Jersey: Prentice Hall.

Hull, J. (2012). Options, Futures and other Derivatives (Global Edition). Pearson Education Limited 8va edición.

Jones, P., Masson, S. y Rosenfeld, E. (1984). Contingent Claims Analysis of Corporate Capital Structure: An Empirical Analysis. Journal of Finance, 39, 611-625.

Kodukula, P. y Chandra, P. (2006). Project Valuation using Real Options: A practiioner's guide. USA: J Ross Publishing.

Longerstaey, J y Spencer, M. (1996). RiskMetrics Technical Document. RiskMetrics (TM) Morgan Stanley MSCI NY https:/www.msci.com/documents/10199/5915b1014206-4ba0-aee2-3449d5c7e95a.

Maffia, O. (1993). Derecho Concursal. CABA, Argentina: De Palma.

Merton, R. (1974). On the pricing of corporate debt: the risk structure of interest rates. Journal of Finance, 29, 449-470.

Milanesi, G. (2013). Teoría de Opciones: Modelos específicos y aplicaciones para valorar estrategias, activos reales e instrumentos financieros (Primera ed.). Bahía Blanca: Editorial de la Universidad Nacional del Sur. REUN.

Milanesi, G. (2014). Momentos estocásticos de orden superior y la estimación de la volatidad implícita: aplicación de la expansión de Edgeworth en el modelo de Black-Scholes. Estudios Gerenciales(30), 336-342.

Milanesi, G. (2016). Un modelo "naive" de opciones barreras para la predicción de fracasos financieros. Estocástica: Finanzas y Riesgo, 6(2), 159-186.

Milanesi, G, Pesce, G y El Alabi, E. (2016). Firm valuation and default probability through exotic (barrier) options. European Accounting and Management Review, 2(2), 56-76.

Mun, J. (2004). Real Options Analysis: Tools and Techniques for Valuing Strategic Investment and Decisions (1 ed.). New York: Wiley.

Reiz, A y Perlich C. (2007). A Market-Based Framework of Bankruptcy Prediction. Journal of Financial Stability, 3(2), 85-131. 
Rivera, J. (1996). Instituciones del Derecho Concursal. CABA, Argentina: Rubinzal Cunsoni.

Sandin, A y Porporato M. (2007). Corporate bankruptcy prediction models applied to emerging economies: Evidence from Argentina in the years 1991-1998. International Journal of Commerce and Management, 17(4), 295-311.

Smaranda, C. (2014). Scoring functions and bankruptcy prediction models - case study for Romanian companies. Procedia Economics and Finance, 10, 217-226.

Smit, H y Trigeorgis, L. (2004). Strategic Investment: Real Options and Games (1 ed.). New Jersey, Estados Unidos: Princeton University Press.

Trillini, J y Milanesi, G. (2015). La cesación de pagos en los procesos judiciales: Una mirada jurídica a la luz de las finanzas corporativas. Doctrina Societaria y Concursal, XXVII, 21-30.

Van der Hoek, J y Elliot, R. (2006). Binomial models in Finance. New York, United State: Springer Science.

Vasicek, O. (1984). Credit Valuation. WP Moody's KMV http:// www.res s ources-actuarielles.net/EX T/I S F / 1226 .n s f/ 0 / c181fb77ee99d464c125757a00505078/\$FILE/Credit_Valuation.pdf.

Vasicek, O. (2001). EDF credit measure and corporate bond pricing. San Francisco, United State: KMV corporation. 


\section{Anexos}

\begin{tabular}{|c|c|c|}
\hline Sticker & Denominación & Nivel 1 \\
\hline ALUA & Aluar & Industrias Manufactureras \\
\hline CEPU & Central Puerto & Empresa de Electricidad, Gas y Agua \\
\hline COME & Sociedad Comercial Del Plata & Corporativos \\
\hline CRES & Cresud & $\begin{array}{l}\text { Agricultura, Ganadería, Aprovechamiento } \\
\text { Forestal, Pesca y Caza }\end{array}$ \\
\hline $\mathrm{CVH}$ & Cablevision Holding & Información y Comunicaciones \\
\hline DGCU2 & Distribuidora De Gas Cuyana & Empresa de Electricidad, Gas y Agua \\
\hline EDN & Edenor & Empresa de Electricidad, Gas y Agua \\
\hline LOMA (i) & Loma Negra & Industrias Manufactureras \\
\hline METR & MetroGas & Empresa de Electricidad, Gas y Agua \\
\hline MIRG & Mirgor SACIFIA & Industrias Manufactureras \\
\hline PAMP & Pampa Energía & Empresa de Electricidad, Gas y Agua \\
\hline TECO2 & Telecom Argentina & Telecomunicaciones \\
\hline TGNO4 & $\begin{array}{l}\text { Transportadora de Gas del } \\
\text { Norte }\end{array}$ & Empresa de Electricidad, Gas y Agua \\
\hline TGSU2 & $\begin{array}{l}\text { Transportadora de Gas del } \\
\text { Sur }\end{array}$ & Empresa de Electricidad, Gas y Agua \\
\hline TRAN & Transener & Empresa de Electricidad, Gas y Agua \\
\hline TXAR & Ternium Argentina & Industrias Manufactureras \\
\hline TS & Tenaris & Industrias Manufactureras \\
\hline YPFD & $\begin{array}{l}\text { Yacimientos Petrolíferos } \\
\text { Fiscales }\end{array}$ & $\begin{array}{l}\text { Minería, Explotación de Canteras y } \\
\text { Extracción de Petróleo y Gas }\end{array}$ \\
\hline AGRO & Agrometal & Industrias Manufactureras \\
\hline AUSO & Autopistas del Sol & Transportes, Correos y Almacenamiento \\
\hline BOLT & Boldt & $\begin{array}{l}\text { Servicios Profesionales, Científicos y } \\
\text { Técnicos }\end{array}$ \\
\hline CADO & Carlos Casado & $\begin{array}{l}\text { Agricultura, Ganadería, Aprovechamiento } \\
\text { Forestal, Pesca y Caza }\end{array}$ \\
\hline CAPU & Caputo & Construcción \\
\hline CAPX & Capex & Empresa de Electricidad, Gas y Agua \\
\hline CARC & Carboclor & $\begin{array}{l}\text { Minería, Explotación de Canteras y } \\
\text { Extracción de Petróleo y Gas }\end{array}$ \\
\hline CECO2 & Enel Generacion Costanera & Empresa de Electricidad, Gas y Agua \\
\hline CELU & Celulosa Argentina & Industrias Manufactureras \\
\hline CGPA2 & Camuzzi Gas Pampeana & Empresa de Electricidad, Gas y Agua \\
\hline CTIO & Consultatio & Construcción \\
\hline
\end{tabular}




\begin{tabular}{|c|c|c|}
\hline Sticker & Denominación & Nivel 1 \\
\hline DYCA & Dycasa & Construcción \\
\hline FERR & Ferrum & Industrias Manufactureras \\
\hline FIPL & Fiplasto & Industrias Manufactureras \\
\hline GARO & Garovaglio \& Zorraquin & $\begin{array}{l}\text { Agricultura, Ganadería, Aprovechamiento } \\
\text { Forestal, Pesca y Caza }\end{array}$ \\
\hline GBAN & Gas Natural BAN & Empresa de Electricidad, Gas y Agua \\
\hline GCLA & Grupo Clarín & Información y Comunicaciones \\
\hline HARG & Holcim Argentina & Industrias Manufactureras \\
\hline HAVA & Havanna & Industrias Manufactureras \\
\hline INDU & Solvay Indupa (UNIPAR) & Industrias Manufactureras \\
\hline INVJ & Inversora Juramento & $\begin{array}{l}\text { Agricultura, Ganadería, Aprovechamiento } \\
\text { Forestal, Pesca y Caza }\end{array}$ \\
\hline IRCP & Irsa Propiedades Ciales. & Construcción \\
\hline LEDE & Ledesma & Industrias Manufactureras \\
\hline LONG & Longvie & Industrias Manufactureras \\
\hline MOLA & Molinos Agro & Industrias Manufactureras \\
\hline MOLI & Molinos Río de la Plata & Industrias Manufactureras \\
\hline MORI & Morixe Hermanos S.A.C.I. & Industrias Manufactureras \\
\hline OEST & Gr. Concesionario del Oeste & Transportes, Correos y Almacenamiento \\
\hline PATA & La Anónima & Comercio al Por Mayor y al Por Menor \\
\hline PATY & Quickfood & Industrias Manufactureras \\
\hline PREN1 & Papel Prensa & Industrias Manufactureras \\
\hline PSUR & Petrolera del Conosur & Comercio al Por Mayor y al Por Menor \\
\hline RICH & Laboratorios Richmond & Comercio al Por Mayor y al Por Menor \\
\hline RIGO & Rigolleau & Comercio al Por Mayor y al Por Menor \\
\hline SAMI & S.A. San Miguel AGICI y F & $\begin{array}{l}\text { Agricultura, Ganadería, Aprovechamiento } \\
\text { Forestal, Pesca y Caza }\end{array}$ \\
\hline SEMI & Molinos Juan Semino & Industrias Manufactureras \\
\hline TGLT & TGLT SA & Construcción \\
\hline
\end{tabular}

Tabla 1.A: Panel de firmas cotizantes seleccionadas (fuente: elaboración propia) 


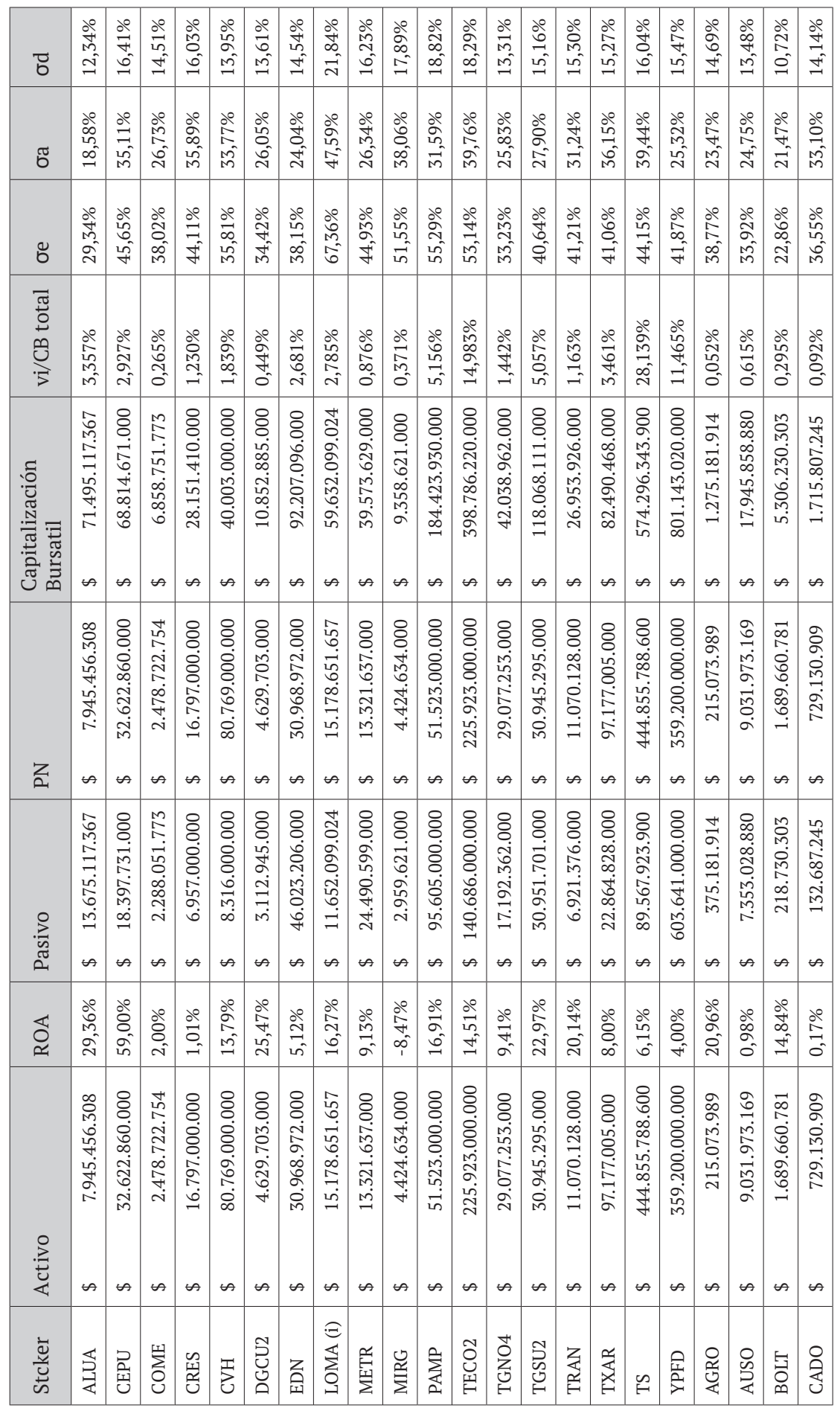




\begin{tabular}{|c|c|c|c|c|c|c|c|c|c|c|c|c|c|c|c|c|c|c|c|c|c|c|c|c|c|}
\hline$\overline{0}$ & & 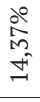 & $\begin{array}{l}\stackrel{0}{\mathrm{~N}} \\
\hat{\mathrm{a}}\end{array}$ & $\begin{array}{l}00 \\
\overrightarrow{0} \\
10 \\
-1\end{array}$ & ఫे & $\begin{array}{c}\stackrel{\partial}{\mathrm{N}} \\
\mathrm{m} \\
-1\end{array}$ & 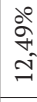 & $\begin{array}{l}\stackrel{\circ}{\sigma} \\
\circ\end{array}$ & $\begin{array}{l}\text { वे } \\
\text { o } \\
\stackrel{2}{a}\end{array}$ & \begin{tabular}{l}
$\stackrel{\circ}{\circ}$ \\
in \\
\multirow{H}{*}{}
\end{tabular} & $\stackrel{\circ}{\circ}$ & $\begin{array}{l}\text { ○े } \\
\text { 今 }\end{array}$ & 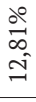 & $\begin{array}{l}\stackrel{\circ}{\vec{m}} \\
\stackrel{\mathrm{m}}{\mathrm{N}}\end{array}$ & $\begin{array}{l}\alpha^{\circ} \\
\sigma^{2} \\
\omega^{2}\end{array}$ & $\begin{array}{l}\text { वें } \\
\text { ज̂ } \\
\end{array}$ & $\begin{array}{l}\stackrel{0}{0} \\
= \\
=\end{array}$ & $\begin{array}{l}\stackrel{\circ}{े} \\
\stackrel{0}{0} \\
\Rightarrow \\
\Rightarrow\end{array}$ & $\begin{array}{l}\stackrel{\circ}{+} \\
\stackrel{+}{S^{\prime}}\end{array}$ & 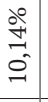 & 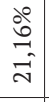 & 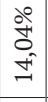 & $\begin{array}{l}\text { वे } \\
\text { d } \\
\text { తi }\end{array}$ & 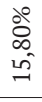 & 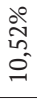 \\
\hline రూ & よે & $\begin{array}{l}\text { वें } \\
\text { mे } \\
\text { ma }\end{array}$ & 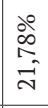 & 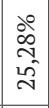 & $\begin{array}{l}\circ \\
0 \\
\text { oे }\end{array}$ & $\mid \begin{array}{l}\circ 0 \\
\infty \\
\infty\end{array}$ & $\begin{array}{c}\text { oे } \\
\text { oे } \\
\text { م }\end{array}$ & \begin{tabular}{l}
$\stackrel{\circ}{\mathrm{N}}$ \\
\multirow{+}{*}{}
\end{tabular} & 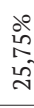 & $\begin{array}{l}\text { Оे } \\
\text { İ } \\
\text { हे }\end{array}$ & $\begin{array}{l}\text { 吕 } \\
\text { hn } \\
\text { s. }\end{array}$ & $\begin{array}{l}\text { ఫें } \\
\text { ô }\end{array}$ & 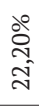 & 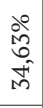 & 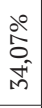 & $\begin{array}{l}\dot{b}^{\circ} \\
\stackrel{1}{0} \\
\dot{m}\end{array}$ & $\begin{array}{l}\stackrel{0}{\sigma} \\
\sigma \\
\sigma \\
\Xi\end{array}$ & 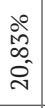 & $\begin{array}{l}\stackrel{0}{0} \\
= \\
=\end{array}$ & $\begin{array}{l}\stackrel{0}{0} \\
\stackrel{0}{=} \\
\exists\end{array}$ & 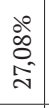 & \begin{tabular}{|}
$\stackrel{\circ}{\circ}$ \\
जे \\
$-\vec{N}$
\end{tabular} & 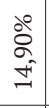 & 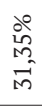 & $\begin{array}{l}\text { m } \\
m^{2}\end{array}$ \\
\hline 0 & \begin{tabular}{l}
$\stackrel{\circ}{\circ}$ \\
\multirow{\infty}{\infty}{}
\end{tabular} & $\begin{array}{l}\text { do } \\
\vdots \\
\text { in }\end{array}$ & $\begin{array}{l}\circ \\
\infty \\
\infty \\
\infty \\
\infty \\
\infty^{\circ}\end{array}$ & \begin{tabular}{c}
$\stackrel{0}{\circ}$ \\
$\stackrel{3}{+}$ \\
\multirow{7}{*}{}
\end{tabular} & $\stackrel{\circ}{\circ}$ & $\begin{array}{l}\stackrel{\circ}{\Delta} \\
\text { 心ิ } \\
\text { mิ }\end{array}$ & 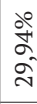 & $\begin{array}{l}\text { ํํㅇ } \\
\text { స్ } \\
\text { స్ }\end{array}$ & $\begin{array}{l}\stackrel{\circ}{2} \\
\text { mf }\end{array}$ & $\begin{array}{l}\stackrel{\circ}{\Xi} \\
0^{\circ}\end{array}$ & $\begin{array}{l}\text { 今े } \\
\text { bे }\end{array}$ & 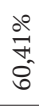 & $\begin{array}{l}\text { 今े } \\
\text { iे } \\
\text { in }\end{array}$ & $\begin{array}{l}\stackrel{\circ}{\mathrm{n}} \\
\text { on } \\
\text { oे }\end{array}$ & 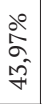 & $\begin{array}{l}\partial^{\circ} \\
\hat{2} \\
0^{-}\end{array}$ & 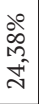 & $\begin{array}{l}\stackrel{\circ}{0} \\
\stackrel{0}{2} \\
\stackrel{2}{N}\end{array}$ & $\begin{array}{l}00 \\
0 \\
0 \\
0 \\
0\end{array}$ & 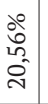 & 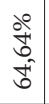 & 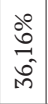 & 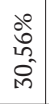 & & 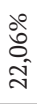 \\
\hline $\begin{array}{l}\bar{\pi} \\
\stackrel{0}{0} \\
0 \\
0 \\
5\end{array}$ & $\begin{array}{l}\stackrel{\circ}{\mathrm{M}} \\
\stackrel{\text { M }}{2}\end{array}$ & 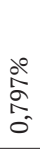 & $\begin{array}{l}\partial 0 \\
\partial \\
0 \\
0\end{array}$ & \begin{tabular}{|l} 
oे \\
. \\
$m$ \\
0 \\
0
\end{tabular} & oे & $\begin{array}{l}\text { ㅇ } \\
0 \\
10 \\
0 \\
0\end{array}$ & $\begin{array}{l}\circ \\
\infty \\
\infty \\
\infty \\
0\end{array}$ & $\begin{array}{l}\text { d̊ㅇ } \\
\text { ठ̊ } \\
0\end{array}$ & 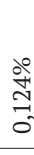 & $\begin{array}{l}\stackrel{\circ}{\circ} \\
\text { ठ̀ } \\
0^{-}\end{array}$ & $\begin{array}{l}\text { ठें } \\
\text { Oे }\end{array}$ & $\begin{array}{l}\stackrel{\circ}{\circ} \\
\stackrel{0}{N} \\
0\end{array}$ & 站 & $\begin{array}{l}\stackrel{\circ}{\circ} \\
\stackrel{-}{=}\end{array}$ & $\begin{array}{l}\text { oे } \\
\text { +े } \\
0 \\
0\end{array}$ & $\begin{array}{c}\text { 今े } \\
\text { Mे } \\
\text { o. }\end{array}$ & $\stackrel{\circ 0}{\vec{m}}$ & $\begin{array}{l}\stackrel{0}{\overrightarrow{7}} \\
\stackrel{2}{a}\end{array}$ & $\begin{array}{l}\text { ळे } \\
\text { mे } \\
0\end{array}$ & $\begin{array}{l}\stackrel{0}{0} \\
\text { ठे } \\
0^{-}\end{array}$ & $\begin{array}{l}\stackrel{0}{1} \\
0 \\
0 \\
0\end{array}$ & $\begin{array}{l}\text { ò } \\
\text { m } \\
\text { + } \\
0\end{array}$ & $\begin{array}{l}\stackrel{0}{a} \\
\overrightarrow{0} \\
0^{-}\end{array}$ & $\begin{array}{l}\text { 우 } \\
\text { î } \\
\text { o. }\end{array}$ & 은 \\
\hline 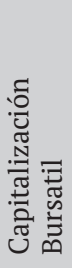 & $\begin{array}{l}8 \\
\varnothing \\
\infty \\
o \\
\stackrel{0}{0} \\
\dot{v} \\
0 \\
\dot{m}\end{array}$ & 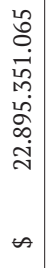 & 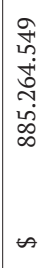 & 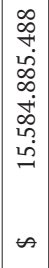 & 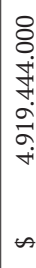 & 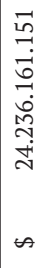 & 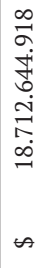 & 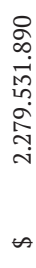 & 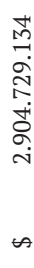 & 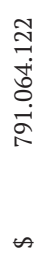 & 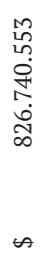 & 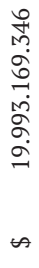 & 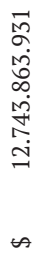 & 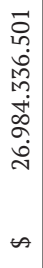 & 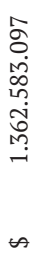 & 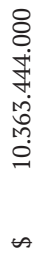 & $\begin{array}{l}n \\
0 \\
0 \\
0 \\
0 \\
0 \\
0 \\
\vdots \\
\vdots \\
a\end{array}$ & 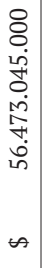 & 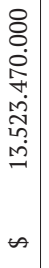 & 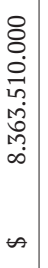 & 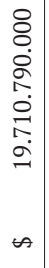 & 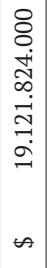 & 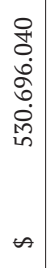 & 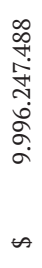 & 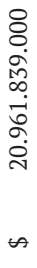 \\
\hline 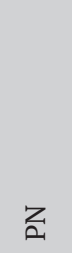 & $\begin{array}{l}8 \\
0 \\
\dot{1}\end{array}$ & 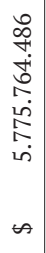 & $\begin{array}{l}\vec{\infty} \\
\infty \\
\stackrel{0}{0} \\
\hat{N} \\
\stackrel{\vec{n}}{.}\end{array}$ & 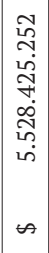 & 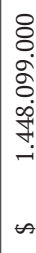 & 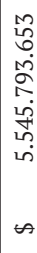 & 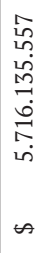 & 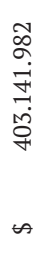 & 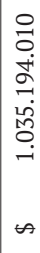 & 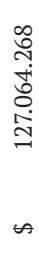 & 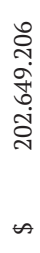 & 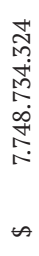 & 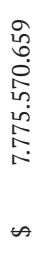 & 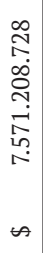 & 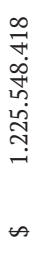 & 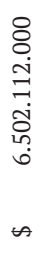 & 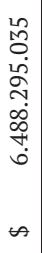 & 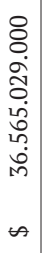 & 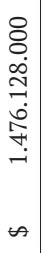 & 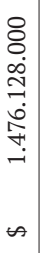 & 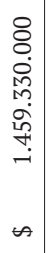 & 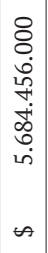 & 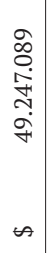 & 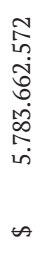 & 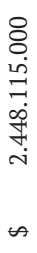 \\
\hline 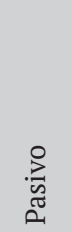 & 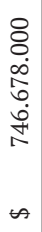 & 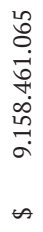 & 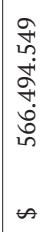 & $\begin{array}{l}\infty \\
\infty \\
0 \\
+1 \\
1 \\
1 \\
10 \\
0 \\
8 \\
0 \\
\sigma \\
\sigma\end{array}$ & 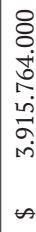 & 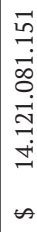 & 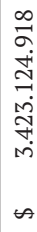 & 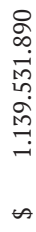 & 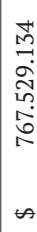 & 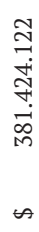 & 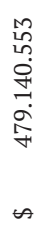 & 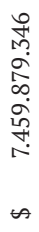 & 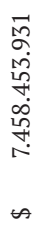 & 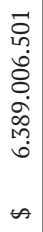 & 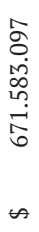 & 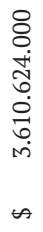 & 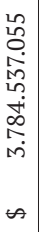 & 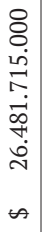 & 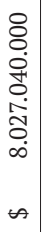 & 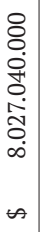 & 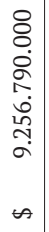 & 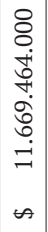 & $\begin{array}{l}9 \\
0 \\
0 \\
0 \\
0 \\
\dot{j} \\
\dot{d} \\
m \\
\infty\end{array}$ & 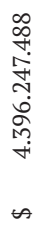 & 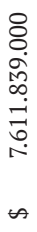 \\
\hline $\begin{array}{l}\text { Ot } \\
\simeq\end{array}$ & $\begin{array}{l}0 \\
0 \\
= \\
=\end{array}$ & सु & $\begin{array}{c}\stackrel{0}{\circ} \\
\underset{\infty}{-1}\end{array}$ & $\begin{array}{l}\text { बें } \\
\text { m. } \\
\text { ले }\end{array}$ & $\begin{array}{l}\stackrel{\circ}{0} \\
\stackrel{0}{0} \\
\infty^{0}\end{array}$ & 产 & $\stackrel{\circ}{\mathrm{f}}$ & $\stackrel{\circ}{\circ}$ & $\begin{array}{l}d \\
\vec{\lambda} \\
\cdots \\
\cdots\end{array}$ & $\begin{array}{l}\text { d̊ } \\
\text { If } \\
\text { of }\end{array}$ & $\begin{array}{l}\text { 今े } \\
\text { m. } \\
n^{2}\end{array}$ & $\stackrel{\substack{0 \\
\rightarrow}}{\rightarrow}$ & $\begin{array}{l}\text { 今े } \\
\text { o } \\
\text { in }\end{array}$ & $\begin{array}{l}\text { iे } \\
\text { अे } \\
0\end{array}$ & 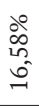 & 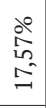 & ○ें & $\begin{array}{l}\stackrel{0}{0} \\
\triangleright \\
i\end{array}$ & ذి & 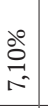 & $\begin{array}{l}\text { वे } \\
0^{0} \\
0^{-}\end{array}$ & $\begin{array}{c}0 \\
\stackrel{\partial}{7} \\
\vdots \\
0\end{array}$ & $\begin{array}{l}\infty \\
\infty \\
\stackrel{+}{0} \\
+1\end{array}$ & $\begin{array}{l}\stackrel{\circ}{\circ} \\
\stackrel{0}{0}\end{array}$ & 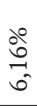 \\
\hline & \begin{tabular}{l}
8 \\
8 \\
$\dot{1}$ \\
\multirow{1}{*}{}
\end{tabular} & 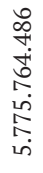 & $\begin{array}{l}\vec{\infty} \\
\infty \\
\stackrel{0}{0} \\
\hat{N} \\
\dot{m} \\
\dot{m}\end{array}$ & 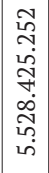 & 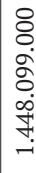 & 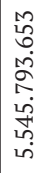 & 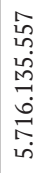 & 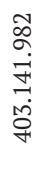 & 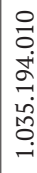 & 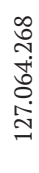 & 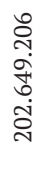 & 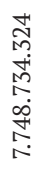 & 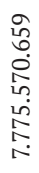 & 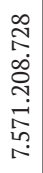 & 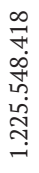 & 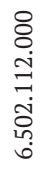 & 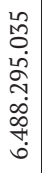 & 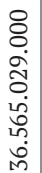 & 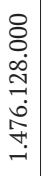 & 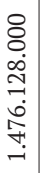 & 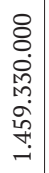 & 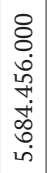 & 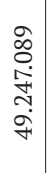 & 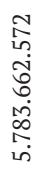 & 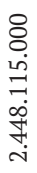 \\
\hline & $\Leftrightarrow$ & $\Leftrightarrow$ & $\infty$ & $\infty$ & $\infty$ & $\infty$ & $\infty$ & $\leftrightarrow$ & $\Leftrightarrow$ & $\infty$ & $\infty$ & $\leftrightarrow$ & $\leftrightarrow$ & $\leftrightarrow$ & $\Leftrightarrow$ & $\infty$ & $\leftrightarrow$ & $\leftrightarrow$ & $\Leftrightarrow$ & $\leftrightarrow$ & $\leftrightarrow$ & $\infty$ & $\infty$ & $\leftrightarrow$ & $\Leftrightarrow$ \\
\hline $\begin{array}{l}\grave{\bar{v}} \\
\bar{u}\end{array}$ & & & 㫕 & $\begin{array}{l}0 \\
\text { પ્પ } \\
\end{array}$ & & . & & ن & & & & 这 & & - & - & - & - & - & - & 旁 & $\begin{array}{l}\mathbb{3} \\
\stackrel{1}{0} \\
\Sigma\end{array}$ & $\stackrel{\overrightarrow{\mathcal{O}}}{\Sigma}$ & $\begin{array}{l}\overrightarrow{\widetilde{z}} \\
\text { 오 }\end{array}$ & 0 & 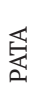 \\
\hline
\end{tabular}




\begin{tabular}{|c|c|c|c|c|c|c|}
\hline$\overline{0}$ & $\begin{array}{l}\stackrel{0}{2} \\
\stackrel{-}{=} \\
0^{-}\end{array}$ & 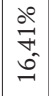 & $\begin{array}{l}\stackrel{0}{\sigma} \\
\overrightarrow{=}\end{array}$ & $\begin{array}{c}\stackrel{0}{0} \\
\dot{m} \\
0 \\
0\end{array}$ & 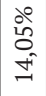 & 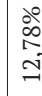 \\
\hline$\tilde{0}$ & 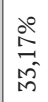 & $\begin{array}{l}\stackrel{\circ}{0} \\
\stackrel{2}{\mathrm{~N}}\end{array}$ & $\begin{array}{l}\stackrel{\circ}{0} \\
\stackrel{+}{+} \\
\infty \\
\stackrel{0}{-}\end{array}$ & $\begin{array}{c}00 \\
0 \\
0 \\
n^{-}\end{array}$ & $\begin{array}{l}\text { do } \\
\text { j+1 } \\
\text { do }\end{array}$ & 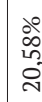 \\
\hline 0 & $\begin{array}{l}\text { oे } \\
\text { bo } \\
\text { i } \\
\text { in }\end{array}$ & 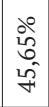 & $\begin{array}{l}\text { oे } \\
\text { bे } \\
\text { ì }\end{array}$ & $\begin{array}{l}\stackrel{0}{\vec{i}} \\
\vec{i}\end{array}$ & $\begin{array}{l}\stackrel{\partial}{\vec{\lambda}} \\
\text { jó } \\
\text { in }\end{array}$ & \\
\hline $\begin{array}{l}\text { चี } \\
0 \\
0 \\
0 \\
y \\
.5\end{array}$ & $\begin{array}{l}\text { o̊ } \\
\text { ఫे } \\
0 \\
0\end{array}$ & $\begin{array}{l}\stackrel{0}{2} \\
0 \\
0 \\
0\end{array}$ & 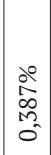 & $\mid \begin{array}{l}0 \\
0 \\
0 \\
0 \\
0 \\
0\end{array}$ & $\begin{array}{l}\text { 워 } \\
\text { M̀ } \\
0^{2}\end{array}$ & 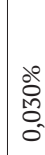 \\
\hline 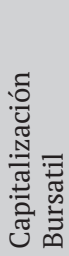 & 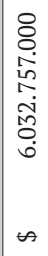 & 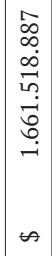 & 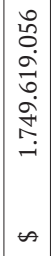 & 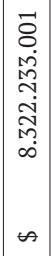 & 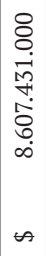 & 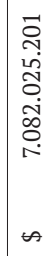 \\
\hline 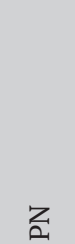 & 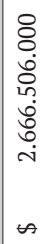 & 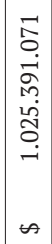 & 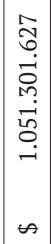 & 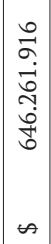 & 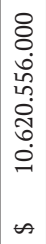 & 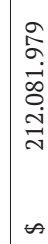 \\
\hline 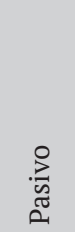 & 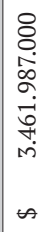 & 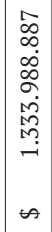 & 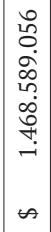 & 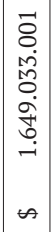 & 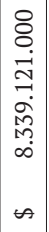 & 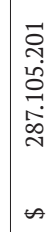 \\
\hline త્ & $\begin{array}{l}\stackrel{0}{0} \\
\text { ํㅗ } \\
\text { î }\end{array}$ & 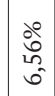 & \begin{tabular}{|l}
20 \\
2 \\
2 \\
$\hat{n}^{2}$
\end{tabular} & 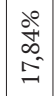 & 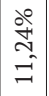 & 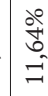 \\
\hline 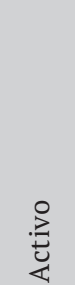 & 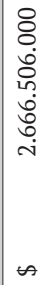 & 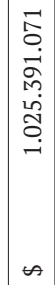 & 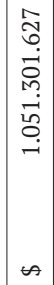 & 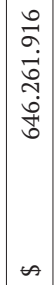 & 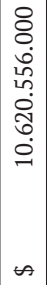 & 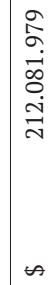 \\
\hline च & 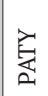 & 愛 & 焉 & 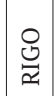 & 䒺 & 1 \\
\hline
\end{tabular}

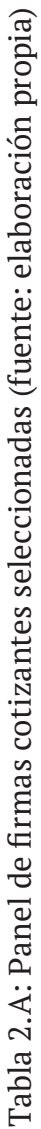




\begin{tabular}{|c|c|c|c|}
\hline Stcker & $\mathrm{N}(\mathrm{d} 1)$ & $\mathrm{N}(\mathrm{d} 2)$ & $\mathrm{BS}=\mathrm{PN}$ \\
\hline ALUA & 0,933022064 & 0,818851606 & $\$ 11.428 .743 .191,66$ \\
\hline CEPU & 0,955344916 & 0,722023425 & $\$ 38.374 .675 .704,13$ \\
\hline COME & 0,943702895 & 0,770736523 & \$ $3.121 .805 .677,28$ \\
\hline CRES & 0,969246942 & 0,768844623 & $\$ 18.850 .368 .277,14$ \\
\hline $\mathrm{CVH}$ & 0,998599176 & 0,972622376 & \$ 69.165.239.093,16 \\
\hline DGCU2 & 0,965728274 & 0,840764058 & $\$ \quad 5.435 .472 .769,29$ \\
\hline EDN & 0,917118327 & 0,734304428 & $\$ 44.213 .411 .764,83$ \\
\hline LOMA (i) & 0,929598412 & 0,487168683 & $\$ 20.508 .039 .373,05$ \\
\hline METR & 0,892146243 & 0,657256357 & $\$ 21.154 .565 .332,71$ \\
\hline MIRG & 0,941695715 & 0,642693889 & \$ $5.468 .597 .365,25$ \\
\hline PAMP & 0,881247948 & 0,572281729 & $\$ 86.891 .379 .388,88$ \\
\hline TECO2 & 0,943993493 & 0,629960503 & $\$ 276.881 .187 .220,48$ \\
\hline TGNO4 & 0,972987325 & 0,86647867 & $\$ 33.400 .937 .773,47$ \\
\hline TGSU2 & 0,934483915 & 0,734935902 & $\$ 40.080 .465 .844,51$ \\
\hline TRAN & 0,956728633 & 0,766057068 & \$ $13.075 .160 .015,39$ \\
\hline TXAR & 0,987485155 & 0,863872492 & $\$ 103.139 .404 .707,15$ \\
\hline TS & 0,987972476 & 0,843535809 & $\$ 469.086 .769 .240,01$ \\
\hline YPFD & 0,902471325 & 0,689756242 & $\$ 543.611 .437 .253,04$ \\
\hline AGRO & 0,906347765 & 0,71787625 & $\$ \quad 324.547 .231,12$ \\
\hline AUSO & 0,958595331 & 0,829460939 & $\$ 10.947 .542 .246,83$ \\
\hline BOLT & 0,999951607 & 0,999358231 & $\$ \quad 1.738 .046 .216,17$ \\
\hline CADO & 0,994610399 & 0,933591287 & $\$ \quad 760.637 .243,79$ \\
\hline CAPU & 0,900406254 & 0,554471988 & $\$ \quad 859.145 .374,48$ \\
\hline CAPX & 0,914879524 & 0,737193419 & \$ 8.389.037.653,05 \\
\hline CARC & 0,7840526 & 0,538668555 & $\$ \quad 229.880 .287,69$ \\
\hline CECO2 & 0,898305316 & 0,681713313 & $\$ \quad 8.549 .338 .246,71$ \\
\hline CELU & 0,872568039 & 0,658646864 & $\$ \quad 2.663 .861 .758,75$ \\
\hline CGPA2 & 0,898807588 & 0,75178192 & $\$ \quad 9.380 .246 .749,18$ \\
\hline CTIO & 0,979065756 & 0,902302346 & $\$ \quad 6.539 .523 .521,32$ \\
\hline DYCA & 0,926961145 & 0,84218138 & $\$ \quad 680.433 .702,12$ \\
\hline FERR & 0,9610363 & 0,82858931 & $\$ 1.236 .271 .802,92$ \\
\hline FIPL & 0,876013488 & 0,694811586 & $\$ \quad 238.234 .097,50$ \\
\hline GARO & 0,921240211 & 0,813315838 & $\$ \quad 323.696 .282,99$ \\
\hline GBAN & 0,917944802 & 0,542312187 & $\$ 10.800 .422 .134,55$ \\
\hline GCLA & 0,957668749 & 0,846631751 & $\$ 9.661 .929 .803,08$ \\
\hline HARG & 0,931829378 & 0,653337335 & $\$ \quad 9.748 .940 .949,88$ \\
\hline
\end{tabular}




\begin{tabular}{|l|l|l|l|l|}
\hline Stcker & $\mathrm{N}(\mathrm{d} 1)$ & $\mathrm{N}(\mathrm{d} 2)$ & \multicolumn{2}{|l|}{$\mathrm{BS}=\mathrm{PN}$} \\
\hline HAVA & 0,958601497 & 0,744545101 & $\$ 1.428 .374 .334,55$ \\
\hline INDU & 0,962395077 & 0,781786776 & $\$ 7.529 .904 .083,18$ \\
\hline INVI & 0,990211886 & 0,957117942 & $\$ 7.349 .355 .732,29$ \\
\hline IRCP & 0,978590531 & 0,914130191 & $\$ 42.821 .294 .033,44$ \\
\hline LEDE & 0,905280749 & 0,826494893 & $\$ 3.417 .200 .399,96$ \\
\hline LONG & 0,905280749 & 0,826494893 & $\$ 3.417 .200 .399,96$ \\
\hline MOLA & 0,813551249 & 0,513831799 & $\$ 4.995 .925 .271,09$ \\
\hline MOLI & 0,902775676 & 0,733824047 & $\$ 8.975 .426 .102,07$ \\
\hline MORI & 0,853609077 & 0,719359002 & $\$$ & $141.350 .671,69$ \\
\hline OEST & 0,944617368 & 0,726836662 & $\$ 7.121 .691 .773,12$ \\
\hline PATA & 0,92864948 & 0,851861046 & $\$ 4.277 .774 .934,14$ \\
\hline PATY & 0,904434373 & 0,601871119 & $\$ 3.914 .843 .388,43$ \\
\hline PREN1 & 0,911678063 & 0,666522867 & $\$ 1.456 .414 .654,74$ \\
\hline RICH & 0,949944072 & 0,855463511 & $\$ 1.413 .240 .629,35$ \\
\hline RIGO & 0,941192897 & 0,871741968 & $\$ 1.038 .009 .500,94$ \\
\hline SAMI & 0,95530487 & 0,805586392 & $\$ 12.869 .764 .380,36$ \\
\hline SEMI & 0,940570839 & 0,818289369 & $\$ 286.114 .812,33$ \\
\hline
\end{tabular}

Tabla 3.A: Parámetros valor del patrimonio neto como opción modelo naive (fuente: elaboración propia) 


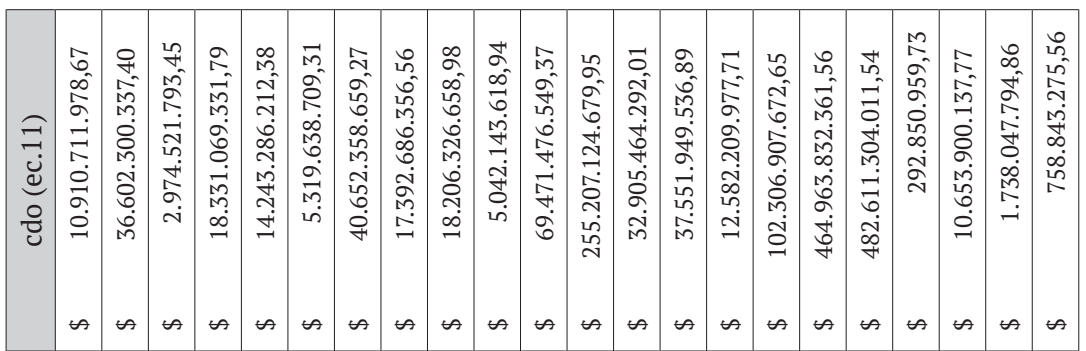

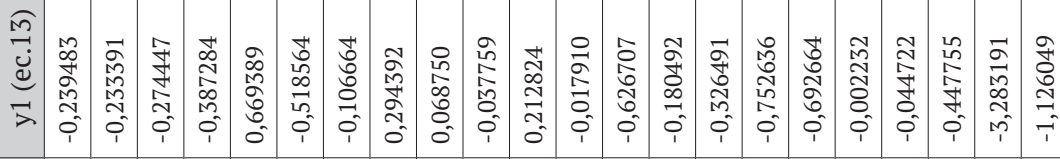

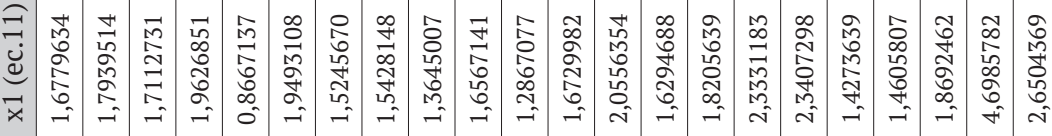

L

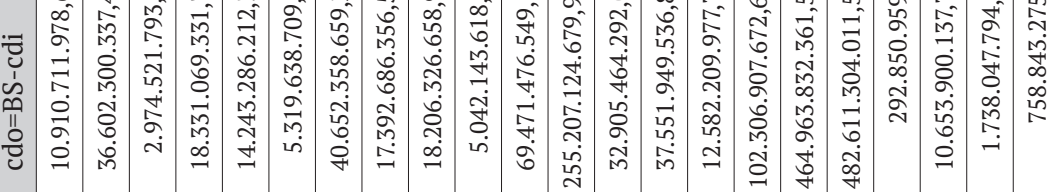

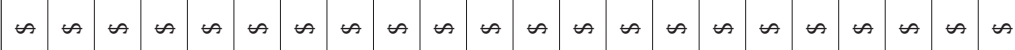

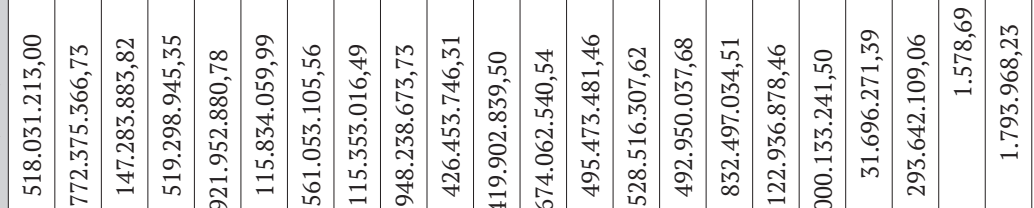

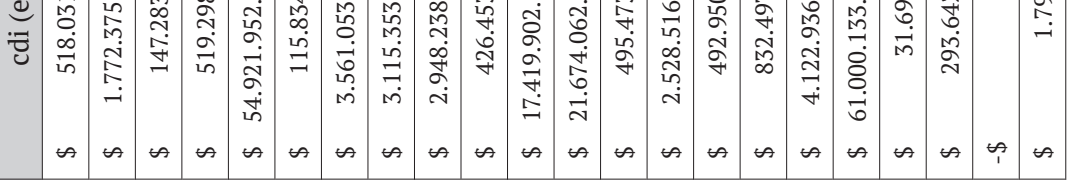

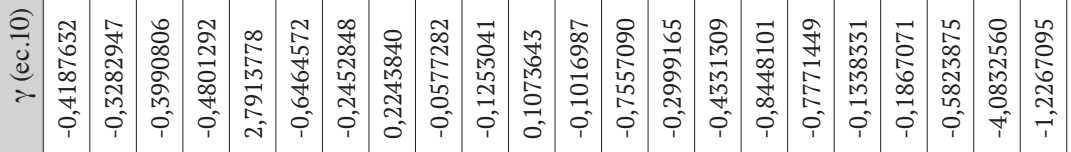

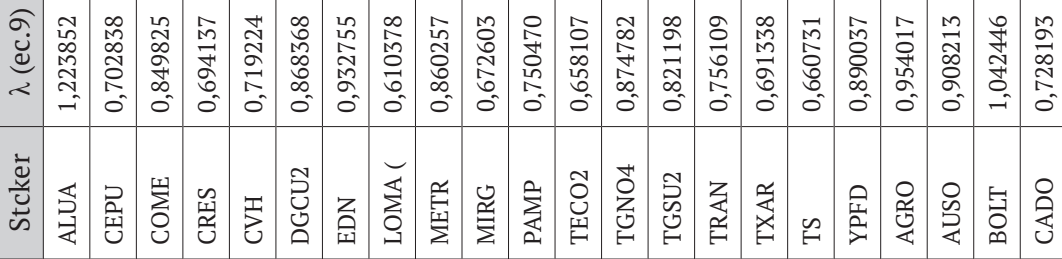




\begin{tabular}{|c|c|c|c|c|c|c|c|c|c|c|c|c|c|c|c|c|c|c|c|c|c|c|c|c|}
\hline 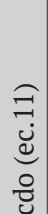 & 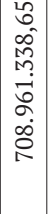 & 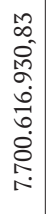 & 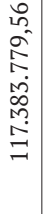 & 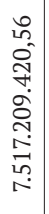 & 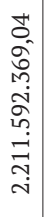 & 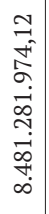 & 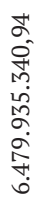 & 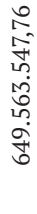 & 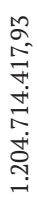 & 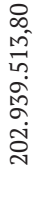 & 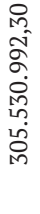 & 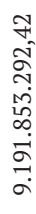 & 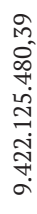 & 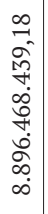 & 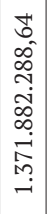 & 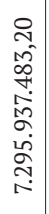 & 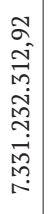 & 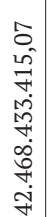 & 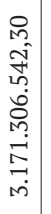 & 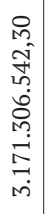 & 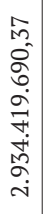 & 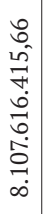 & 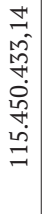 & 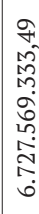 \\
\hline & $\leftrightarrow$ & $\infty$ & $\leftrightarrow$ & $\Leftrightarrow$ & $\leftrightarrow$ & $\leftrightarrow$ & $\leftrightarrow$ & $\Leftrightarrow$ & $\leftrightarrow$ & $\leftrightarrow$ & $\leftrightarrow$ & $\leftrightarrow$ & $\infty$ & $\Leftrightarrow$ & $\infty$ & $\leftrightarrow$ & $\infty$ & $\infty$ & $\leftrightarrow$ & $\Leftrightarrow$ & $\leftrightarrow$ & $\leftrightarrow$ & $\leftrightarrow$ & $\theta$ \\
\hline 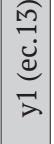 & 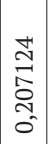 & $\begin{array}{l}\overrightarrow{+} \\
0 \\
0 \\
0 \\
0 \\
1\end{array}$ & 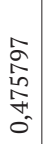 & $\begin{array}{l}\infty \\
\stackrel{M}{=} \\
\vec{\delta} \\
0 \\
0^{\prime}\end{array}$ & $\begin{array}{l}\frac{0}{2} \\
\vec{m} \\
\overrightarrow{0} \\
0\end{array}$ & \begin{tabular}{l}
$\stackrel{0}{0}$ \\
\multirow{+}{0}{} \\
0 \\
0 \\
0 \\
1
\end{tabular} & 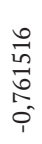 & $\begin{array}{l}\text { oे } \\
0 \\
0 \\
\text { I } \\
0 \\
1\end{array}$ & 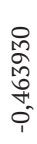 & 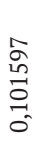 & 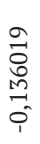 & 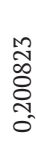 & $\begin{array}{l}\text { nn } \\
\text { Oे } \\
0 \\
+ \\
0 \\
1\end{array}$ & 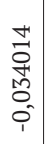 & 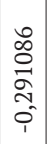 & 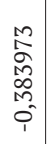 & 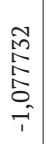 & 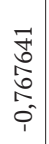 & 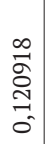 & $\begin{array}{l}\infty \\
\overrightarrow{0} \\
\stackrel{0}{0} \\
\overrightarrow{0}\end{array}$ & 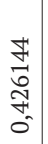 & 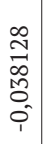 & 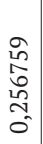 & 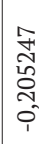 \\
\hline 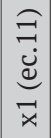 & 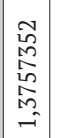 & 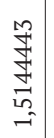 & $\begin{array}{l}n \\
\alpha \\
\alpha \\
\infty \\
o \\
\alpha \\
\sigma\end{array}$ & 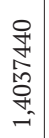 & 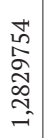 & 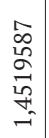 & 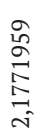 & $\begin{array}{l}\vec{D} \\
\text { ర్ } \\
\infty \\
0 \\
0 \\
-\end{array}$ & 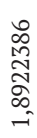 & \begin{tabular}{l} 
J \\
\multirow{H}{*}{} \\
$\infty$ \\
$M$ \\
-
\end{tabular} & \begin{tabular}{l}
8 \\
8 \\
8 \\
\multirow{+}{+}{} \\
$\underset{-1}{0}$
\end{tabular} & 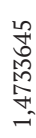 & 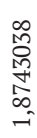 & 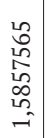 & 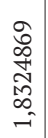 & 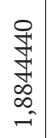 & 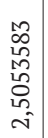 & 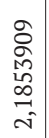 & 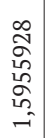 & 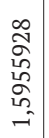 & 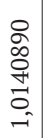 & 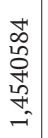 & 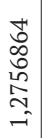 & 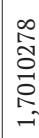 \\
\hline 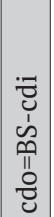 & $\begin{array}{l}n \\
0 \\
0 \\
0 \\
0 \\
0 \\
0 \\
o \\
0 \\
0 \\
0\end{array}$ & 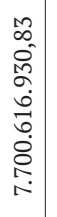 & 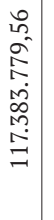 & 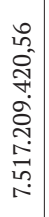 & 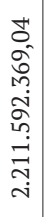 & 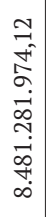 & 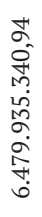 & 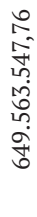 & 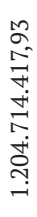 & 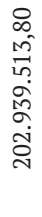 & 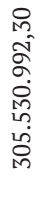 & 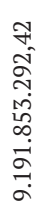 & 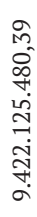 & 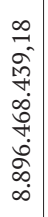 & 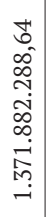 & 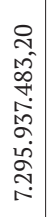 & 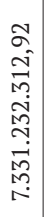 & 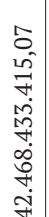 & 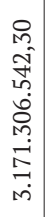 & 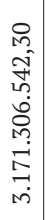 & 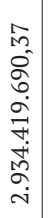 & 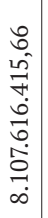 & 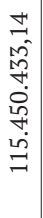 & 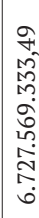 \\
\hline & $\Leftrightarrow$ & $\leftrightarrow$ & $\Leftrightarrow$ & $\leftrightarrow$ & $\leftrightarrow$ & $\leftrightarrow$ & $\leftrightarrow$ & $\Leftrightarrow$ & $\leftrightarrow$ & $\infty$ & $\leftrightarrow$ & & $\infty$ & $\leftrightarrow$ & $\infty$ & $\Leftrightarrow$ & $\Leftrightarrow$ & $\infty$ & $\Leftrightarrow$ & $\leftrightarrow$ & $\leftrightarrow$ & $\leftrightarrow$ & $\leftrightarrow$ & $\infty$ \\
\hline
\end{tabular}

m $⿻$ ส

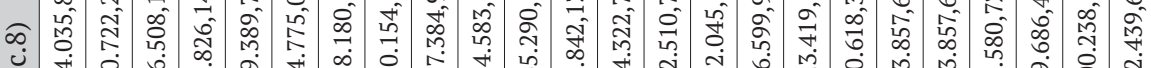

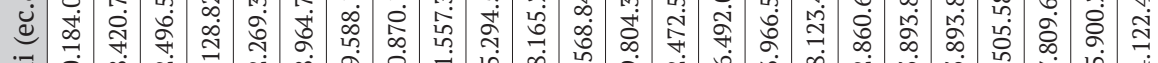

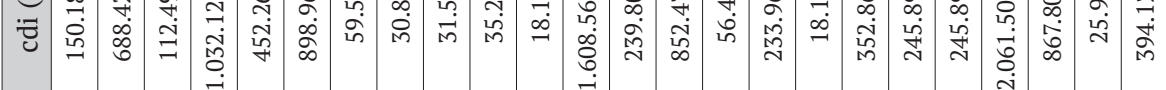

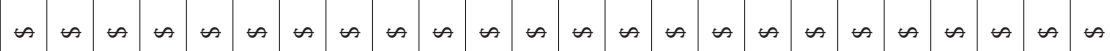

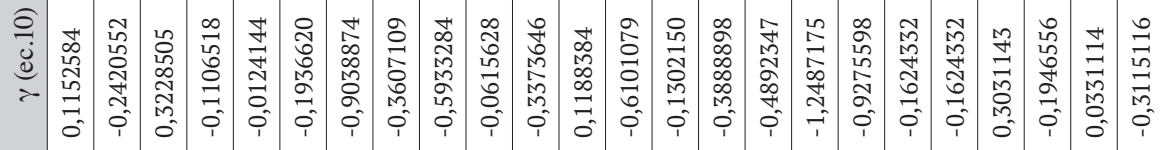

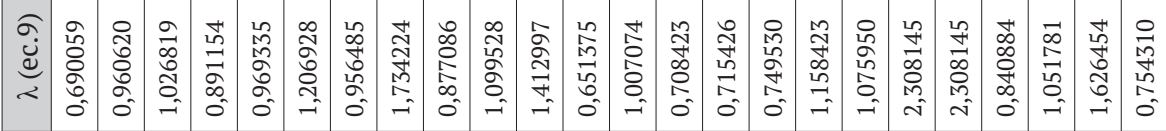

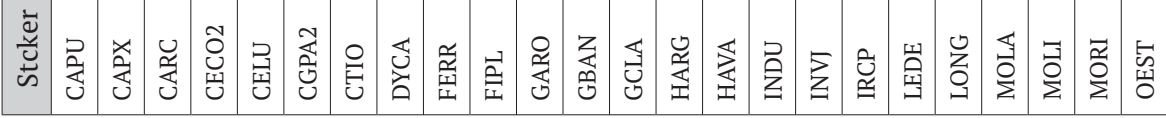




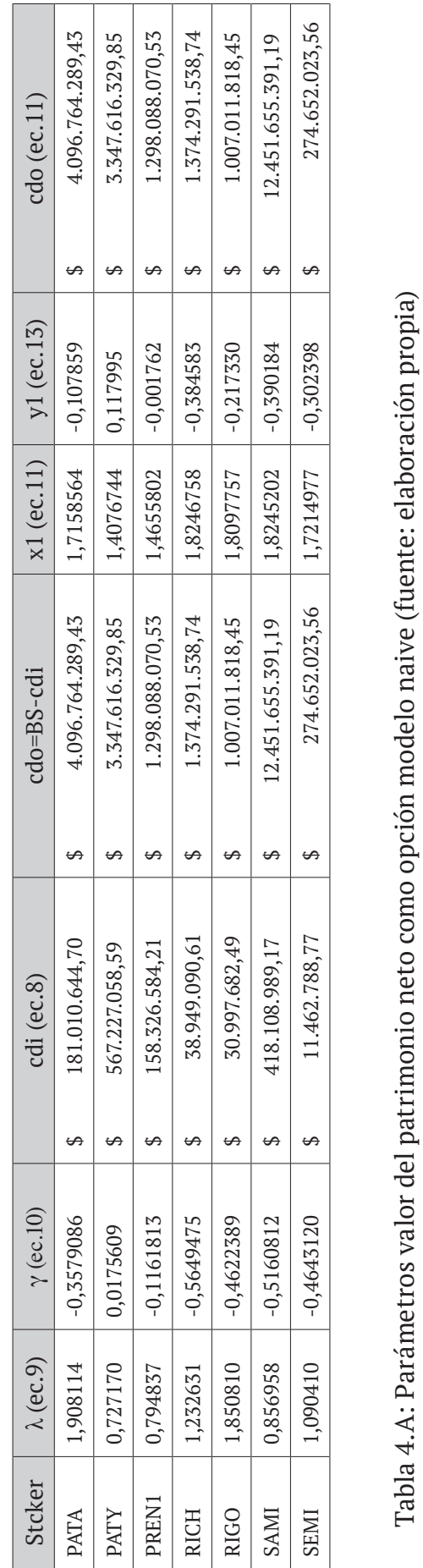




\begin{tabular}{|c|c|c|c|c|}
\hline Stcker & PD (ec.16) & PD (ec.6) & VD (total) (ec.18) & VD (total) (ec.17) \\
\hline ALUA & $0,01 \%$ & 0,000002118\% & $\$ 8.697 .083,94$ & $\$ 50,84$ \\
\hline CEPU & $0,01 \%$ & $0,000000680 \%$ & $\$ 4.436 .426,47$ & $\$ 13,70$ \\
\hline COME & $38,68 \%$ & $24,757882399 \%$ & $\$ 2.652 .739 .454,06$ & $\$ 4.505 .596,85$ \\
\hline CRES & $39,72 \%$ & $27,287262881 \%$ & $\$ 11.180 .675 .883,44$ & $\$ 94.513 .204,83$ \\
\hline $\mathrm{CVH}$ & $86,03 \%$ & $0,145188578 \%$ & \$ 34.416.354.983,09 & $\$ 1.068 .358,89$ \\
\hline DGCU2 & $0,13 \%$ & $0,007674600 \%$ & $\$ 14.049 .259,00$ & $\$ 3.742,39$ \\
\hline EDN & $32,35 \%$ & $16,590729510 \%$ & $\$ 29.830 .476 .654,28$ & $\$ 410.139 .284,05$ \\
\hline LOMA (i) & $42,57 \%$ & $18,862271166 \%$ & $\$ 25.385 .239 .861,77$ & $\$ 313.289 .151,08$ \\
\hline METR & $30,65 \%$ & $11,489115300 \%$ & $\$ 12.130 .098 .662,10$ & $\$ 39.809 .992,19$ \\
\hline MIRG & $82,19 \%$ & $70,731953486 \%$ & $\$ 7.691 .528 .706,06$ & $\$ 24.589 .554,15$ \\
\hline PAMP & $24,90 \%$ & $5,211113517 \%$ & $\$ 45.921 .436 .754,06$ & $\$ 495.523 .636,04$ \\
\hline TECO2 & $24,01 \%$ & $9,901305301 \%$ & $\$ 95.768 .218 .384,89$ & $\$ 5.916 .052 .253,63$ \\
\hline TGNO4 & $5,71 \%$ & $2,526122779 \%$ & $\$ 2.399 .488 .964,30$ & $\$ 15.317 .378,33$ \\
\hline TGSU2 & $1,90 \%$ & $0,160109001 \%$ & $\$ 2.246 .448 .129,97$ & $\$ 9.560 .032,44$ \\
\hline TRAN & $2,93 \%$ & $0,601457118 \%$ & $\$ 790.436 .697,69$ & $\$ 1.885 .271,79$ \\
\hline TXAR & $10,15 \%$ & $5,712998527 \%$ & $\$ 8.372 .275 .878,16$ & $\$ 163.121 .784,90$ \\
\hline TS & $15,84 \%$ & $9,649793310 \%$ & \$ 90.966.091.043,07 & $\$ 15.594 .217 .163,91$ \\
\hline YPFD & $44,48 \%$ & $24,736805886 \%$ & $\$ 356.312 .778 .239,77$ & $\$ 22.721 .460 .715,71$ \\
\hline AGRO & $2,45 \%$ & $0,108999780 \%$ & $\$ 31.225 .452,87$ & $\$ 726,19$ \\
\hline AUSO & $33,46 \%$ & $22,441304161 \%$ & $\$ 6.004 .657 .100,35$ & $\$ 24.764 .831,34$ \\
\hline BOLT & $0,00 \%$ & $0,000023551 \%$ & $\$ 1.253,07$ & $\$ 3,69$ \\
\hline CADO & $14,28 \%$ & $10,014556828 \%$ & $\$ 244.949 .694,41$ & $\$ 157.915,62$ \\
\hline CAPU & $43,30 \%$ & $18,760066571 \%$ & $\$ 1.312 .355 .932,18$ & $\$ 753.982,60$ \\
\hline CAPX & $9,66 \%$ & $2,215180583 \%$ & $\$ 2.211 .277 .041,10$ & $\$ 4.044 .416,70$ \\
\hline CARC & $63,88 \%$ & $18,116525822 \%$ & $\$ 565.513 .385,35$ & $\$ 29.678,13$ \\
\hline $\mathrm{CECO} 2$ & $0,92 \%$ & $0,003865991 \%$ & $\$ 142.870 .489,22$ & $\$ 2.023,17$ \\
\hline CELU & $31,71 \%$ & $10,650918407 \%$ & $\$ 1.559 .956 .631,43$ & $\$ 305.287,75$ \\
\hline CGPA2 & $0,18 \%$ & $0,000054134 \%$ & $\$ 42.668 .193,98$ & $\$ 77,04$ \\
\hline CTIO & $22,64 \%$ & $15,790100716 \%$ & $\$ 4.237 .204 .882,44$ & $\$ 26.225 .571,43$ \\
\hline DYCA & $4,11 \%$ & $0,872131617 \%$ & $\$ 93.726 .457,89$ & $\$ 13.156,60$ \\
\hline FERR & $3,82 \%$ & $1,177286941 \%$ & $\$ 110.942 .880,83$ & $\$ 42.427,18$ \\
\hline FIPL & $25,19 \%$ & $7,589629163 \%$ & \$ 199.271.992,71 & $\$ 14.277,29$ \\
\hline GARO & $0,94 \%$ & $0,042496182 \%$ & $\$ 7.731 .395,29$ & $\$ 70,89$ \\
\hline GBAN & $21,88 \%$ & $5,378246121 \%$ & $\$ 4.374 .821 .974,99$ & $\$ 7.823 .452,27$ \\
\hline GCLA & $13,32 \%$ & $6,741553465 \%$ & $\$ 1.696 .872 .379,71$ & $\$ 2.636 .032,88$ \\
\hline HARG & $29,11 \%$ & $13,134587983 \%$ & $\$ 7.856 .460 .723,45$ & $\$ 42.374 .831,54$ \\
\hline
\end{tabular}




\begin{tabular}{|l|l|l|l|l|}
\hline Stcker & PD (ec.16) & PD (ec.6) & VD (total) (ec. 18$)$ & VD (total) (ec. 17$)$ \\
\hline HAVA & $7,69 \%$ & $2,472024183 \%$ & $\$ 104.767 .577,66$ & $\$ 13.511,54$ \\
\hline INDU & $4,05 \%$ & $1,117822662 \%$ & $\$ 420.105 .736,26$ & $\$ 454.122,71$ \\
\hline INVI & $6,29 \%$ & $4,143050803 \%$ & $\$ 574.754 .320,35$ & $\$ 1.177 .372,79$ \\
\hline IRCP & $0,00 \%$ & $0,000047791 \%$ & $\$ 1.420 .737,97$ & $\$ 469,89$ \\
\hline LEDE & $7,44 \%$ & $1,468558770 \%$ & $\$ 1.005 .877 .133,75$ & $\$ 633.681,76$ \\
\hline LONG & $7,44 \%$ & $1,468558770 \%$ & $\$ 622.078 .761,36$ & $\$ 23.990,39$ \\
\hline MOLA & $70,35 \%$ & $32,540398574 \%$ & $\$ 13.866 .271 .893,09$ & $\$ 38.924 .265,53$ \\
\hline MOLI & $55,66 \%$ & $37,655055990 \%$ & $\$ 10.643 .948 .904,35$ & $\$ 31.149 .958,48$ \\
\hline MORI & $89,84 \%$ & $79,889140137 \%$ & $\$ 476.794 .652,28$ & $\$ 46.521,36$ \\
\hline OEST & $50,99 \%$ & $35,539968874 \%$ & $\$ 5.096 .822 .415,12$ & $\$ 11.549 .231,90$ \\
\hline PATA & $8,24 \%$ & $2,779528540 \%$ & $\$ 1.727 .329 .180,64$ & $\$ 4.515 .364,52$ \\
\hline PATY & $75,04 \%$ & $56,962097934 \%$ & $\$ 4.527 .013 .194,95$ & $\$ 5.128 .331,18$ \\
\hline PREN1 & $38,23 \%$ & $19,188748257 \%$ & $\$ 635.169 .251,70$ & $\$ 60.619,74$ \\
\hline RICH & $11,17 \%$ & $5,218907472 \%$ & $\$ 195.415 .463,99$ & $\$ 353.727,05$ \\
\hline RIGO & $0,03 \%$ & $0,000130417 \%$ & $\$ 2.881 .985,95$ & $\$ 1,69$ \\
\hline SAMI & $7,72 \%$ & $2,830370070 \%$ & $\$ 664.175 .000,02$ & $\$ 960.975,14$ \\
\hline SEMI & $4,21 \%$ & $1,034591515 \%$ & $\$ 298.128 .211,84$ & $\$ 22.067,12$ \\
\hline
\end{tabular}

Tabla 5.A: Probabilidades de insolvencia modelo opciones barreras - naive y valor esperado de las probabilidades de insolvencia (fuente: elaboración propia) 